Submitted to Astrophysical Journal June 24, 2015

\title{
Charting the Interstellar Magnetic Field causing the Interstellar Boundary Explorer (IBEX) Ribbon of Energetic Neutral Atoms
}

\author{
P. C. Frisch \\ Dept. Astronomy and Astrophysics, University of Chicago, Chicago, IL 60637 \\ A. Berdyugin and V. Piirola \\ Finnish Centre for Astronomy with ESO, University of Turku, Finland \\ A. M. Magalhaes and D. B. Seriacopi \\ Inst. de Astronomia, Geofisica e Ciencias Atmosfericas, Universidade de Sao Paulo, Brazil \\ S. J. Wiktorowicz \\ Dept. Astronomy, University of California at Santa Cruz, Santa Cruz, CA \\ B-G Andersson \\ SOFIA Science Center, USRA, Moffett Field, CA \\ H. O. Funsten \\ Los Alamos National Laboratory, Los Alamos, NM \\ D. J. McComas ${ }^{1}$ \\ Southwest Research Institute, San Antonio, TX \\ N. A. Schwadron \\ Space Science Center, University of New Hampshire \\ J. D. Slavin \\ Harvard-Smithsonian Center for Astrophysics, Cambridge, MA
}

A. J. Hanson

${ }^{1}$ Also University of Texas, San Antonio, TX 
School of Informatics and Computing, Indiana University, Bloomington, IN

C.-W. Fu

School of Computer Engineering, Nanyang Technological University, Singapore

\begin{abstract}
The interstellar magnetic field (ISMF) near the heliosphere is a fundamental component of the solar galactic environment that can only be studied using polarized starlight. The results of an ongoing survey of the linear polarizations of local stars are analyzed with the goal of linking the interstellar magnetic field that shapes the heliosphere to the nearby field in interstellar space. We present new results on the direction of the magnetic field within $40 \mathrm{pc}$ obtained from analyzing polarization data using a merit function that determines the field direction that provides the best fit to the polarization data. Multiple magnetic components are identified, including a dominant interstellar field, $\mathrm{B}_{\mathrm{POL}}$, that is aligned with the direction $\ell, b=36.2^{\circ}, 49.0^{\circ}\left( \pm 16.0^{\circ}\right)$. Stars tracing $\mathrm{B}_{\mathrm{POL}}$ have the same mean distance as stars that do not trace $\mathrm{B}_{\mathrm{POL}}$, but show weaker average polarizations consistent with a smaller column density of polarizing material. $\mathrm{B}_{\mathrm{POL}}$ is aligned with the ISMF traced by the IBEX Ribbon to within $7.6_{-7.6}^{+14.9}$ degrees. The variations in the polarization position angle directions derived from the data that best match $\mathrm{B}_{\mathrm{POL}}$ indicate a low level of magnetic turbulence, $\sim 9^{\circ} \pm 1^{\circ}$. The direction of $\mathrm{B}_{\mathrm{POL}}$ is obtained after excluding polarization data tracing a separate magnetic structure that appears to be associated with interstellar dust deflected around the heliosphere. The velocities of local interstellar clouds relative to the local standard of rest (LSR) increase with the angles between the LSR velocities and $\mathrm{B}_{\mathrm{POL}}$, indicating that the kinematics of local interstellar material is ordered by the ISMF. The Loop I superbubble that extends close to the Sun contains dust that reddens starlight and whose distance is determined by the color excess $\mathrm{E}(\mathrm{B}-$ $\mathrm{V}$ ) of starlight. Polarizations caused by grains aligned with respect to $\mathrm{B}_{\mathrm{POL}}$ are consistent with the location of the Sun in the rim of the Loop I superbubble. An angle of $76.8_{-27.6}^{+23.5}$ between $\mathrm{B}_{\mathrm{POL}}$ and the bulk LSR velocity the local interstellar material indicates a geometry that is consistent with an expanding superbubble. The efficiency of grain alignment in the local ISM has been assessed using stars where both polarization data and hydrogen column density data are available. Nearby stars appear to have larger polarizations than expected based on reddened sightlines, which is consistent with previous results, but uncertainties are
\end{abstract}


large. Optical polarization and color excess $\mathrm{E}(\mathrm{B}-\mathrm{V})$ data indicate the presence of nearby interstellar dust in the BICEP2 field. Color excess E(B-V) indicates an optical extinction of $A_{\mathrm{V}} \sim 0.59$ in the BICEP2 field, while the polarization data indicate that $A_{\mathrm{V}}>0.09 \mathrm{mag}$. The IBEX Ribbon ISMF extends to the boundaries of the BICEP2 region.

Subject headings: ISM: clouds, dust, magnetic fields - Physical processes: polarization - Sun: heliosphere

\section{Introduction}

The Sun is traveling through a dynamically evolving interstellar environment that contains low density, magnetized, partially ionized interstellar material traveling rapidly away from the center of the Loop I superbubble. The heliosphere is shaped by the solar wind interaction with the interstellar gas and magnetic field. A unique diagnostic of the direction of the magnetic field at the heliosphere location is provided by the Ribbon of energetic neutral atoms (ENAs) discovered by the Interstellar Boundary Explorer (IBEX, McComas et al. 2009; Schwadron et al. 2009; Funsten et al. 2009, 2013; Schwadron et al. 2011). The flux of $\mathrm{TeV}$ galactic cosmic rays at the Earth is controlled by the ISMF direction that is traced by the IBEX Ribbon (Schwadron et al. 2014). Similarly, galactic cosmic ray fluxes onto exoplanets and their astrospheres (stellar wind bubbles) depend on the interstellar magnetic field that surrounds the exoplanet system (Frisch 1993). Understanding the role of the interstellar magnetic field (ISMF) in the past and future galactic environments of the solar system and nearby exoplanet systems requires knowledge of the magnetic field configuration and its connection to the magnetized and partially ionized medium in the galactic neighborhood of the heliosphere. The purpose of this study is to connect the ISMF that shapes the heliosphere with the extended magnetic field in the nearby interstellar clouds.

Studies over the past century of optical and ultraviolet (UV) interstellar absorption lines, and the reddening of starlight by interstellar dust, have revealed the physical properties, dynamics and distribution of local interstellar material within 40 pc of the Sun (Frisch et al. 2011). Although the Sun was known to be located in a region of very low interstellar dust densities (Fitzgerald 1968), measurements of polarized starlight proved to be a viable method for testing the very local interstellar magnetic field (Piirola 1977; Tinbergen 1982). Nevertheless, the interstellar dust content and magnetic field configuration close to the heliosphere have been enigmatic. In this paper we present new high-sensitivity measurements of polarized starlight that allow filling the gaps in our knowledge of the configuration of the local interstellar magnetic field within 40 pc of the Sun. 
Polarized starlight provides the only method for tracing the ISMF direction in the low density interstellar medium (ISM) near the solar system. For polarization due to dichroic extinction, starlight becomes linearly polarized while traversing a medium formed by charged asymmetric submicron-sized interstellar dust grains that are aligned with the most opaque grain axis oriented perpendicular to the interstellar magnetic field (Hoang \& Lazarian 2014). Comparisons between polarized dust emission, polarized synchrotron emission, and linearly polarized optical starlight indicate that optical polarization position angles are oriented parallel to the ISMF direction in the nearby ISM (Frisch et al. 2015b). The low interstellar column densities near the Sun (Bohlin et al. 1978; Frisch \& York 1983; Wood et al. 2005) impose two conditions on the study of the local ISMF: high-sensitivity polarization measurements are required, and the analysis method must utilize statistically-weighted data so that low-significance polarization data can be included.

The goal of charting the direction of the ISMF within $40 \mathrm{pc}$ is to connect the interstellar field in the solar neighborhood with the interstellar magnetic field that shapes the heliosphere. In Frisch et al. (2010a, Paper I) we derived the orientation of the local ISMF by assuming that the field has a dipole configuration, and that the pole of this field could be retrieved by applying a minimization algorithm to the polarization position angles. The analysis was based on polarization data in the literature. New data were collected on the polarizations of nearby stars in order to fully the constrain the magnetic field direction (Frisch et al. 2012, Paper II). Applying a minimization procedure with statistically weighted position angles, to the extended data set produced a interstellar field direction close to the direction of the IBEX Ribbon ISMF. The two directions were within $\sim 33^{\circ} \pm 27^{\circ}$ of each other. The velocity vector of the bulk flow of the cluster of local interstellar clouds (CLIC) relative to the Local Standard of Rest (LSR) was found to form an angle of $\sim 76^{\circ}$ with the best-fitting ISMF to the polarization data. Subsequently, we identified a distinct nearby magnetic structure that appears to be formed by aligned dust grains entrained in the interstellar magnetic field draping over the heliosphere (Frisch et al. 2015a).

The present study includes new data that have been collected in both the northern and southern hemispheres in order to map the magnetic field in nearby interstellar space, described in Section $(\S)$ 2. We refine the analysis method used in Papers I and II where a merit function is used to establish the best-fitting ISMF direction to the ensemble of interstellar polarization measurements (\$3). The best-fitting ISMF direction to all qualifying polarization data is given in \$4.1. Polarization data associated with a distinct filamentary polarization structure are identified (\$4.2). Omission of the filament stars from the data sample gives a best-fitting ISMF direction in close agreement with the IBEX ISMF direction (\$4.3) although not all significant polarizations agree (\$4.4). Magnetic turbulence is estimated from the dispersion of polarization position angles (\$4.5). Additional possible 
unrecognized local ISMF components are briefly considered (\$4.6). The broad implications of these results are discussed in \$5, including the relation between $\mathrm{B}_{\mathrm{POL}}$ and the IBEX Ribbon (\$5.1), galactic cosmic ray asymmetries (\$5.2), the origin of the separate polarization filament (\$5.3), the gas-dust relationship (\$5.4), and local interstellar clouds and the Loop I superbubble $(\$ 5.5)$. The polarization data show that nearby low-extinction interstellar dust, and magnetic fields, are found in the BICEP2 region of study (\$5.6). Conclusions are presented in $\$ 6$. Appendices provide additional details on the stars with polarization position angles that best match the IBEX ISMF direction that traces the LIC field (Appendix A), the source of the color excess data (Appendix $\mathrm{B}$ ), the formulae that characterize the efficiency of polarization mechanisms (Appendix $(\mathrm{C})$ ), and the conversion of heliocentric velocities to the LSR for the purpose of comparing the cloud velocity with the ISMF direction (Appendix D). As an aside, it is found that the contemporary solar apex motion is similar to that found by Herschel (1783, Appendix D).

\section{Polarization data used to determine the magnetic field direction}

Starlight polarization attributed to interstellar dust aligned with respect to the magnetic field was discovered in 1949 (see Andersson 2015, for a review). Multiple measurements of starlight polarized in the interstellar medium were acquired during the last half of the 20th century, and later assembled into a single catalog (Heiles 2000). The 20th century data sets used in this analysis included the discovery of the interstellar magnetic field within $40 \mathrm{pc}$ of the Sun in the fourth galactic quadrant $\left(\ell=270^{\circ}-360^{\circ}\right.$, Tinbergen 1982; Piirola 1977). More recently, high sensitivity polarimeters capable of $3 \sigma$ detections of polarization strengths $<0.01 \%$ have become available (Piirola et al. 2014; Berdyugin et al. 2014; Perevra \& Magalhães 2007; Wisniewski et al. 2007; Bailey et al. 2010, 2015; Wiktorowicz \& Nofi 2015; Wiktorowicz et al. 2015).

Modern polarimeters are capable of detecting interstellar polarizations in the low column density nearby clouds. Surveys of interstellar polarizations indicate that, in the absence of line-of-sight depolarization, polarization strengths and extinction are related (Serkowski et al. 1975; Fosalba et al. 2002, \$5.4, Appendix C). A heuristic relation corresponding to the upper envelope of the distribution of polarization strength as a function of color-excess E(B-V) provides a nominal upper-limit for expected polarizations. For the low column densites of the ISM within $25 \mathrm{pc}, \mathrm{N}\left(\mathrm{H}^{\mathrm{o}}\right)<10^{18.7}$ (Wood et al. 2005), polarizations of up to $\sim 0.014 \%$ are expected (see the discussion of the relation between $\mathrm{E}(\mathrm{B}-\mathrm{V})$, polarization strengths, and $N(\mathrm{H})$ in Appendix $\mathrm{C}$ ). Modern polarimeters are therefore capable of mapping the direction of the local ISMF. 
In this paper we utilize new polarization measurements that have been acquired with the DiPol2 polarimeter at the KVA telescope in La Palma, Canary Islands (Berdvugin et al. 2014), the IAGPOL polarimeter at the LNA at Picos dos Dios in Brazil (Pereyra \& Magalhães 2007; Wisniewski et al. 2007), and the POLISH2 polarimeter at the Lick Observatory in California (Wiktorowicz \& Nofi 2015; Wiktorowicz et al. 2015). These data include measurements with $3 \sigma$ sensitivities of $0.01 \%$ or better. Data on the polarizations of nearby stars from the high-sensitivity survey with the PlanetPol instrument (Bailey et al. 2010), the Loop I polarization survey of Santos et al. (2011), and the Heiles (2000) polarization catalog are also incorporated into the polarization database used in this study.

The region of study in this paper is restricted to stars within $90^{\circ}$ of the heliosphere nose since this interval includes the patch of nearby polarizing dust grains found by Tinbergen (1982), it includes the region of right ascension RA> 17 HR where Bailey et al. (2010) have shown that polarization strengths increase with distance, and it contains the IBEX Ribbon ( $(1)$. This region also includes the star $\alpha$ Oph (14 pc) with striking properties for the foreground ISM (Munch \& Unsold 1962; Frisch 1981; Frisch et al. 1987). The angular constraint that program stars must be located within $90^{\circ}$ of the heliosphere nose effectively restricts stars to the galactic center hemisphere, but includes stars at high-latitudes.

These combined data form a heterogeneous set of over 700 measurements of polarizations for 520 stars within 40 pc. Three hundred of these stars are within $90^{\circ}$ of the direction of the heliosphere nose, $\ell, b=3.2^{\circ}, 15.5^{\circ}$ (e.g. McComas et al. 2015). Fig. 1 histograms the distribution of the angles between the stars in the database and the heliosphere nose as a function of the significance of the polarization measurement and the epoch of measurement. Thirty percent of these stars within $40 \mathrm{pc}$ have polarizations that have been measured at a significance of $P / \Delta P \geq 2.0$, where $P$ and $\Delta P$ are polarization and the mean error of the polarization. The merit function that we have developed to assess the best-fitting ISMF direction to these polarization data includes a weighting factor that allows the use of measurements at all levels of $P / \Delta P$ in this analysis so that weaker polarizations are useful data points (\$3). The data from the 20th century provide an unbiased spatial sampling of the magnetic field (thin gray line in Fig. 11), whereas many of the 21st century data were collected specifically for this project and were selected by their proximity to the heliosphere nose.

Polarizations are plotted against the star distance in Fig. 2 for those stars that are located between $\mathrm{RA}=17 \mathrm{H}$ and $\mathrm{RA}=22 \mathrm{H}$ in the first galactic quadrant, $\ell=0^{\circ}-90^{\circ}$. Bailey et al. (2010) showed that the strengths of polarizations in this region increase with distance (also see Fig. 7 in Paper II), and we have shown that this increase is consistent with a dust bridge reaching from the solar vicinity out to the North Polar Spur region (Frisch et al. 2015b). 
The search for the ISMF direction that best matches these polarization data uses polarization position angles, $\theta_{\mathrm{PA}}$, and not polarization strengths (\$3). Polarization position angles that trace a single magnetic field direction are independent of the wavelength of the measurement and therefore provide a consistently defined quantity for data collected at different sensitivities using different spectral bands. In addition, the size distributions of the polarizing grains are poorly known. In situ measurements of interstellar dust grains by Ulysses and other spacecraft show that the size range extends from $\sim 0.04-2.0 \mu \mathrm{m}$ if the grains are compact silicates (Frisch et al. 1999; Landgraf et al. 2000; Krueger et al. 2014; Sterken et al. 2015). Since the wavelength of maximum polarization strengths depend on grain sizes, composition, and porosity (Serkowski et al. 1975; Andersson \& Potter 2006; Andersson 2015), the polarization strengths can not be compared because of the different spectral bands of the various data sets.

The polarization position angles of the data assembled for this analysis are mapped in Fig. 3. The uncertainties on the polarization position angles, $\arctan (\Delta P / 2 P)$ where $\Delta P$ is the uncertainty on the polarization strength, are plotted with "fan-shaped" symbols. The angular width of the fan indicates the uncertainty on the polarization position angle. The dots show stars where $P / \Delta P<2.0$. A nominal limit of $P / \Delta P=2.0$ is used for plotting position angles since interstellar polarizations of stars within $40 \mathrm{pc}$ tend to be weak with fewer stars showing $P / \Delta P>3$. The analysis (93) incorporates data with all uncertainty levels using the appropriate weighting factor. Since the mean errors of the polarizations from the different data sets vary, it is possible to have two measurements of the same star, corresponding to two adjacent data points in Fig. 3, that show different mean errors. With only a few exceptions, these types of adjacent points do not represent discrepancies but rather result from the use of early polarization data with large uncertainties. Generally all measurements of a star are included with the use of weighted data points, with the exception of a few older data points that are clearly superceded by more accurate recent data. The circled stars in Fig. 3 show the locations of the stars that trace a separate nearby magnetic structure, or "filament" ( $\$ 4.2, \$ 5.3$, Frisch et al. 2015a). The filament runs roughly parallel to the northern border of the IBEX Ribbon of energetic neutral atoms (ENAs, McComas et al. 2009; Schwadron et al. 2011), the brightest parts of which are plotted in yellow.

The data used in this paper differ from that of Paper II in that this analysis utilizes new observations from the LNA and KVA observatories (Table 2), and Lick Observatory (Wiktorowicz et al. 2015), and excludes otherwise qualifying Heiles data if later higherquality data are also available. 


\section{Merit function for deriving the ISMF direction from polarization data}

Due to the low extinction of the nearby ISM where interstellar polarization strengths are weak, we have developed a method for combining weighted polarization data to evaluate the best-fitting ISMF direction to those data, utilizing all data including where $P / \Delta P<$ 2.0. The strategy is to search for a regular dipole-like component to the ISMF that best matches the total group of polarization position angles indicated by the polarization data. A merit function describes how well the polarization position angles are aligned with the field direction. It is evaluated for each possible ISMF direction to find the minimum value that provides the best-fitting ISMF direction for the polarization dataset.

The merit function utilizes the fact that for linearly polarized starlight aligned with the direction of the ISMF in the diffuse interstellar medium the sine of the polarization position angle 1 will be zero in a coordinate system that is aligned with the ISMF poles. In Paper I and II, and here, the star sample is restricted to stars within $40 \mathrm{pc}$ and $90^{\circ}$ of the heliosphere nose. The same strategy of minimizing the sine of polarization position angles to evaluate the local ISMF direction is used also, where all possible ISMF directions are tested in order to determine the ISMF direction. In Paper I the data sample was restricted to $P / \Delta P>2.5$ (where the polarization is $P$ and the mean error of the measurement is $\Delta P$ ). The limit of $P / \Delta P>2.5$ resulted in the omission of a large amount of useful data. In Paper II, using a larger set of data that included new measurements, weighting was introduced into the merit function that tested for the best ISMF direction, so that data points with low statistical significance could still be incorporated into the analysis.

This use of a weighting function is continued in the present analysis. The weighting function is based on the bivariate statistical description of polarization position angles given in Naghizadeh-Khouei \& Clarke (1993, NKC). This probability distribution accounts for the fact that while the polarizations are always positive, the underlying Stokes parameters can be either positive or negative. The outcome of using the NKC weighting function is that the statistical likeliness of position angles in the wings of the distribution are increased over the expectation of a true Gaussian distribution, and this property increases the value of incorporating polarizations with $P / \Delta P<2.0$ into the analysis. Above $P / \Delta P=6$ the probability distribution for polarization position angles reverts to a Gaussian.

The merit function $F_{\mathrm{II}}\left(\mathrm{B}_{\mathrm{i}}\right)$ that tests for the ISMF direction that best describes the ensemble of polarization position angles, then becomes a combination of the requirement that

\footnotetext{
${ }^{1}$ The polarization position angle is defined as the angle between the linear polarization vector and a north-south meridian passing through the star, with values in the interval $0^{\circ}$ and $180^{\circ}$ and increasing toward the east.
} 
the mean sine of the position angle is minimized and the statistical weighting of polarization position angles is maximized:

$$
F_{\mathrm{II}}\left(B_{\mathrm{i}}\right)=\mathrm{N}^{-1} \sum_{\mathrm{n}=1}^{\mathrm{N}} f_{\mathrm{n}}\left(B_{\mathrm{i}}\right) \text { where } f_{\mathrm{n}}\left(B_{\mathrm{i}}\right)=\left|\frac{\sin \left(\theta_{\mathrm{n}}\left(B_{\mathrm{i}}\right)\right)}{G_{\mathrm{n}}}\right|
$$

The contribution of each individual star $n$ to $F_{\mathrm{II}}\left(\mathrm{B}_{\mathrm{i}}\right)$, for ISMF direction $B_{\mathrm{i}}$, is $f_{\mathrm{n}}\left(B_{\mathrm{i}}\right)$. The quantity $\theta_{\mathrm{n}}\left(B_{\mathrm{i}}\right)$ is the polarization position angle $\theta_{\mathrm{PA}, \mathrm{n}}$ for star $\mathrm{n}$, which is calculated with respect to the $\mathrm{i}^{\text {th }}$ possible interstellar magnetic field direction $B_{\mathrm{i}}$. The sum is over $N$ stars. $G_{\mathrm{n}}$ (eqn. 2) is the NKC weighting factor for each star:

$$
G_{\mathrm{n}}\left(\theta_{\mathrm{obs}} ; \theta_{\mathrm{o}}, P_{\mathrm{o}}\right)=\frac{1}{\sqrt{\pi}}\left\{\frac{1}{\sqrt{\pi}}+\eta_{\mathrm{o}} \exp \left(\eta_{\mathrm{o}}^{2}\right)\left[1+\operatorname{erf}\left(\eta_{\mathrm{o}}\right)\right]\right\} \exp \left(-\frac{\mathrm{P}_{\mathrm{o}}^{2}}{2}\right)
$$

for observed position angle $\theta_{\mathrm{obs}}$, "true" position angle $\theta_{\mathrm{o}}, P_{\mathrm{o}}=\frac{P_{\text {true }}}{\sigma}$, mean error $\sigma=d P$, $\eta_{\mathrm{o}}=\frac{P_{\mathrm{o}}}{\sqrt{2}} \cos \left[2\left(\theta_{\mathrm{obs}}-\theta_{\mathrm{o}}\right)\right]$, and the Gaussian error function $\operatorname{erf}(Z)=\frac{2}{\sqrt{\pi}} \int_{0}^{Z} \exp \left(-t^{2}\right) d t$.

Two limits were imposed in order to prevent unrecognized properties of the data from biasing the result. The value of $G_{\mathrm{n}}$ was capped at 3.5 in the analysis program to prevent overweighting any single star in the analysis. By use of this cap, the effects of possibly unrecognized intrinsically polarized stars, or or any single data set with systematically smaller mean errors (e.g. the PlanetPol data), are minimized. A second limit was imposed by requiring $G_{\mathrm{n}}$ to be $1 \times 10^{-5}$ or larger. Experience showed that weights below this level represent insignificant data points, but potentially cause numerical problems in the code. Note that polarization strengths are not used in this analysis because of the diversity of spectral bands with which these data were acquired (\$2).

The possible directions for the ISMF pole, $B_{\mathrm{i}}$, are then tested over a grid of one-degree intervals on the sky. For the best comparison between the interstellar polarization data and the ISMF traced by the IBEX Ribbon, only stars within $40 \mathrm{pc}$ of the solar system and $90^{\circ}$ of the heliosphere nose are included in the evaluation. The ISMF direction determined from all qualifying data is denoted $B_{\mathrm{ALL}}$ (see \$4.1).

The function $F_{\mathrm{II}}\left(\mathrm{B}_{\mathrm{i}}\right)$ does not incorporate the variance of the array being minimized. We have therefore also tested a merit function with the additional term, $\sigma\left(F_{\mathrm{II}}\left(B_{\mathrm{i}}\right)\right)$, corresponding to the standard deviation of the array $F_{\mathrm{II}}\left(\mathrm{B}_{\mathrm{i}}\right)$, for the function that is being minimized:

$$
F_{\text {III }}\left(B_{\mathrm{i}}\right)=F_{\mathrm{II}}\left(B_{\mathrm{i}}\right) \times \sigma\left(F_{\mathrm{II}}\left(B_{\mathrm{i}}\right)\right)
$$

We make the assumption that if the method for determining the ISMF direction is to yield a robust result, then the directions found from eqs. 1 and 3 must be similar. This condition 
is satisfied. The use of eqn. 3 does increase the spatial gradients of the merit function, affecting the uncertainties of the $B_{\mathrm{ALL}}$. Results from eqn. 3 are not used to determine the best-fitting ISMF to these polarization data.

\section{Direction of ISMF within $40 \mathrm{pc}$}

\subsection{Best-fitting local magnetic field direction to all data}

The simplest approximation is to assume that there is a single ISMF that controls the optical interstellar polarizations for stars within $40 \mathrm{pc}$ and $90^{\circ}$ of the heliosphere nose, as was also assumed in Papers I and II. All of the qualifying observations are used in the evaluations of the ISMF direction, except for the 20th century data collected in the Heiles (2000) catalog where observations range in precision levels. Those data are not used if a more recent, and presumably more precise, measurement is available for the same star. Justification for the approximation of a single field direction is provided by the low column densities, $\mathrm{N}\left(\mathrm{H}^{\circ}\right)<10^{18.7}$, of the ISM within $\sim 25 \mathrm{pc}$ (e.g. Wood et al. 2005), and the fact that local clouds flow through space with roughly similar velocity vectors in the LSR, suggesting a common origin for the clouds (Frisch et al. 2002, 2011). It is shown below, however, that multiple magnetic structures appear to be present.

Analyzing the qualifying set of polarization data (\$2), with the merit function $F_{\mathrm{II}}\left(\mathrm{B}_{\mathrm{i}}\right)$ (eqn. (1) based on weighted data points (eqn. 2), gives a best-fitting ISMF direction that is toward the direction $\ell=16.3^{\circ}, b=27.0^{\circ}$ (Fig. \&, left). The uncertainty on this direction is determined by the width of the minimum of $F_{\mathrm{II}}\left(\mathrm{B}_{\mathrm{i}}\right)$. Fig. 5 , left, shows the value of $F_{\mathrm{II}}\left(\mathrm{B}_{\mathrm{i}}\right)$ plotted against the angle from this best-fitting ISMF direction for each location in the sky. The uncertainty on the best-fitting ISMF direction is assumed to be the angle that clearly distinguishes the minimum of $F_{\mathrm{II}}\left(\mathrm{B}_{\mathrm{i}}\right)$ from an adjacent secondary minimum, or $\pm 15^{\circ} \mathrm{cm}^{-2}$. This new best-fitting direction differs by $27.6 \pm 29.2^{\circ}$ from the result of Paper II, which is not a significant difference. A test was also made to determine whether the use of $F_{\mathrm{III}}\left(\mathrm{B}_{\mathrm{i}}\right)$ changed the best-fitting ISMF direction from these data, and it gave a similar direction directed toward $\ell=15.3^{\circ}, b=27.0^{\circ}$.

\subsection{Identifying a filamentary-shaped magnetic structure}

As the sky coverage of the underlying polarization dataset improves, it becomes more likely that inhomogeneities in the direction of the local ISMF will be sampled. Since the purpose of this study is to connect the ISMF that shapes the heliosphere with the local 
interstellar field, the analysis of the best-fitting ISMF to the polarization data needs to take into account the possibility there are multiple local ordered components of the magnetic field, so that polarizations clearly associated with a magnetic field that is different from the one that shapes the heliosphere can be omitted from the fits. In this section we identify a clearly identifiable secondary magnetic structure. The properties of this filament suggest that it is related to interstellar dust grains deflected around the heliosphere (Frisch et al. 2015a). In this section we justify the selection of these polarizations as belonging to a separate magnetic structure, and in the following section the magnetic field direction in the local ISM is evaluated for a data set that omits data that trace the polarization filament.

Using data from the PlanetPol polarimeter, Bailey et al. (2010) showed that the polarization strengths for stars in the region $\mathrm{RA}>17 \mathrm{H}$ increase with the distance of the target star. In Paper II we showed that the stars that formed the upper envelope in the polarization versus distance relation for this subset of the PlanetPol data contains a group of stars within 40 pc that traces a magnetic structure with an ordered ISMF direction that extends to within $10 \mathrm{pc}$ of the Sun. In Paper II, this ordered field was characterized by a position angle gradient of $\mathrm{PA}_{\mathrm{RA}}$ of $\sim-0.25$ degrees per parsec (based on the fit $\theta_{\mathrm{RA}}=36.0( \pm 1.4)-0.25( \pm 0.03) D$ for distance $D$ and position angles expressed in the equatorial coordinate system, $\theta_{\mathrm{RA}}$, e.g. with respect to right ascension).

Given this evidence for a magnetic structure suggested by the upper envelope to the polarization vs. distance relation for the PlanetPol data, we have searched for additional stars within $40 \mathrm{pc}$ in this spatial interval that might also show polarization position angles that vary systematically with distance indicating an ordered magnetic field. A total of thirteen stars (HD 131977, HD 161797, HD 120467, HIP 82283, HD 119756, HD 144253, HD 130819, HD 161096, HD 134987, HD 136894) were identified in the current data set by a systematic decrease of $\theta_{\text {gal }}$ with distance (see below). The stars tracing the magnetic structure are located between 6 pc and 29 pc from the Sun and appear to form an elongated feature spanning an angle of $\sim 5^{\circ} \times 98^{\circ}$, where the polarization position angles are parallel to the axis of the structure (see the polarization data points that are circled in Fig. 3). The geometric configuration of this structure is filamentary or edge-on. Since a filament would occupy the smallest volume of space, we suggest that it is filamentary.

The gradient in $\theta_{\text {gal }}$ with distance is quantified by dividing the thirteen stars into two separate groups and performing a linear fit to the variation of polarization vs. distance for each group of stars. A slightly different slope of the $\theta_{\mathrm{PA}}$ vs. distance relationship was obtained for the two groups (Fig. 6). The most slowly varying ISMF component is traced by seven stars and includes the original three PlanetPol stars from Paper II. The linear fit to $\theta_{\mathrm{PA}}$ vs. distance for the first set gives $\theta_{\text {gal }}=106.8( \pm 1.5)-0.53( \pm 0.08) D_{\text {star }}$, with a reduced 
$\chi^{2}$ of 1.217 (lower group of stars in Fig. 6). The second set of six stars in the same extended filamentary-shaped feature can be fit by the line $\theta_{\text {gal }}=130.0( \pm 15.2)-0.68( \pm 0.65) D_{\text {star }}$, with a reduced $\chi^{2}$ of 0.528 (upper group of stars in Fig. 6). More than thirteen data points are plotted in Fig. 6 because several of these stars have been observed multiple times.

The angle found in Paper II for the rotation of polarization position angles with distance was $\sim-0.25^{\circ}$ per parsec for position angles presented in the equatorial coordinate system, whereas the slopes in the galactic coordinate system are $-0.53^{\circ}$ to -0.68 degrees per parsec, and the stars span an angular range of $\sim 98^{\circ}(\$ 4.2)$. The factor of at least two difference between the slopes suggests that neither the galactic coordinate system nor the equatorial coordinate system is the correct system for evaluating the characteristics of the magnetic structure traced by the filament stars. It appears as if some of the variation of the polarization position angle with distance is, instead, due to the rotation of the coordinate systems over the angular interval spanned by the star positions.

To remove this bias introduced by the coordinate system, we have applied the analysis method of $\$ 3$ to the filament stars. Evaluating the minimum of $F_{\mathrm{II}}\left(\mathrm{B}_{\mathrm{i}}\right)$ for the filamentstar data gives a magnetic field direction for the filament, $B_{\mathrm{FIL}}$, toward $\ell=359^{\circ}$, and $b=19^{\circ}$. A better direction for the magnetic field direction traced by the filament stars is found in Frisch et al. (2015a, see Table 1), which incorporated new polarization measurements of three additional stars not used here. The polarization position angles expressed with respect to the pole $B_{\text {FIL }}$ do not vary systematically with the distance of the star. Evidently the distance dependence of the polarization position angles defined by $\theta_{\text {gal }}(\$ 4.2)$ is partly due to the rotation of the galactic coordinate system over the $90^{\circ}$ span of the filament.

Fig. 6, right, shows the polarization position angle that is calculated with respect to the filament magnetic field direction, $B_{\mathrm{FIL}}$, and plotted against the angular distance between the star and the end of the filament. The filament end is defined by star HD 172167, located at $\ell, b=67^{\circ}, 19^{\circ}$. The steady variation of the polarization angle $\mathrm{PA}_{\text {filament }}$ along the filament length suggests that $B_{\text {FIL }}$ provides a better coordinate system for expressing filament polarizations than does the north galactic pole (or north terrestrial pole). The best-fitting ISMF direction to the filament stars, from eqn. 1, is directed toward the heliosphere nose defined by the inflow velocity vector of neutral interstellar He into the heliosphere. The magnetic turbulence associated with the filament polarizations is $\pm 9.6^{\circ}$, based on the harmonic mean of the measurement uncertainties and the dispersion of the polarization position angles with respect to the filament magnetic field direction. The polarization position angles of the filament are obviously not consistent with the local ISMF direction obtained in Paper II, or with the new fit to the entire data set in this paper (Fig. 4, left). 


\subsection{Best-fitting local magnetic field direction without filament polarizations}

Since the filament polarizations appear to define an isolated magnetic structure (see previous section), the fitting process has been repeated for a data set that is identical to that used to obtain $B_{\mathrm{ALL}}$ except that the thirteen stars that trace the filament polarizations are omitted. The results of the fit performed with the omission of the filament stars are shown in Fig. 4, right, and the uncertainties on that fit are shown in Fig. 5, right. For this new fit, the best-fitting ISMF direction is toward $\ell=36.2^{\circ}, b=49.0^{\circ}$. Comparison of the distribution of $F_{\mathrm{II}}\left(\mathrm{B}_{\mathrm{i}}\right)$ for the evaluations with and without the filament stars (Fig. 4), clearly shows that the merit function obtained from the star sample that omits the filament stars is more clearly defined than the irregularly shaped minimum of the merit function that is based on the entire data sample. The uncertainty on this best-fitting ISMF direction is assumed to occur where $F_{\mathrm{II}}\left(\mathrm{B}_{\mathrm{i}}\right)$ is $10 \%$ larger than the minimum, giving an uncertainty on this direction of $\pm 16^{\circ}$ (Fig. 5, right). The directions of $\mathrm{B}_{\mathrm{POL}}$ and the IBEX ISMF, $\mathrm{B}_{\mathrm{IBEX}}$, are the same to within the uncertainties (Table 1).

\subsection{Statistical properties of the merit function for the dominant ISMF, $\mathrm{B}_{\mathrm{POL}}$}

At first glance, the excellent agreement between the ISMF direction obtained from the polarization data $\mathrm{B}_{\mathrm{POL}}$ after stars tracing a separate magnetic structure are omitted from the sample and the ISMF traced by the IBEX Ribbon almost seems too good to be correct. Since other unrecognized magnetic features may be present in these data, and the volume of space sampled by the polarization data is large, it is remarkable that the local ISMF field direction found from the polarization data is so close to the ISMF indicated by the IBEX Ribbon (Table 1). We therefore look more closely at the values of the individual parameters in eqn. 1 to determine whether all of the polarization data with $P / \Delta P>2.0$ are tracing $\mathrm{B}_{\mathrm{POL}}$, as opposed to $\mathrm{B}_{\mathrm{POL}}$ being traced by only a subset of the polarization data.

The properties of the polarization position angles referenced to the best-fitting ISMF direction, $\mathrm{B}_{\mathrm{POL}}$, are viewed from two perspectives: (i) The three-dimensional statistical properties of $F_{\mathrm{II}}\left(\mathrm{B}_{\mathrm{i}}\right)$ as a function of the probability $G_{\mathrm{n}}$, sine $\left(\theta_{\mathrm{POL}}\right.$, and $F_{\mathrm{II}}\left(\mathrm{B}_{\mathrm{i}}\right)$ evaluated for the polarization position angles calculated with respect to $\mathrm{B}_{\mathrm{POL}}$. (ii) The probability of the position angle (eqn. 22) versus the position angle of the star with respect to $\mathrm{B}_{\mathrm{POL}}$.

For the first approach, the statistical characteristics of the data set that yields the best-fitting ISMF can be represented by plotting the individual components of the merit function, $F_{\mathrm{II}}\left(\mathrm{B}_{\mathrm{i}}\right)$ (eqn. [1, Fig. 7), where the best-fitting ISMF direction $\mathrm{B}_{\mathrm{POL}}$ corresponds to the minimum value of $F_{I I}\left(B_{\mathrm{i}}\right)$. The function $f_{\mathrm{n}}\left(B_{\mathrm{i}}\right)$ (eqn. 1) achieves low values for either 
high-probability polarization position angles $\left(G_{\mathrm{n}}\right.$, eqn. 2) or small $\sin \left(\theta_{\mathrm{n}}\right)$. Fig. 7 shows the $F_{\mathrm{II}}\left(\mathrm{B}_{\mathrm{i}}\right)$ plotted against the probability $G_{\mathrm{n}}$ that the data point traces $\mathrm{B}_{\mathrm{POL}}$, and the sine of the position angle $\sin \left(\theta_{\mathrm{n}, \mathrm{i}}\right)$ calculated with respect to the best-fitting ISMF $B_{\mathrm{n}}=\mathrm{B}_{\mathrm{POL}}$. The probability shows the probability $G_{\mathrm{n}}$ (eqn. 2) for each star $i$, normalized to a maximum value of one. The right-hand axis labeled "sin(theta)" shows the sine of the polarization position angle for each star in the coordinate system defined with respect to the best-fitting ISMF at the north pole 2 The vertical axis labeled "merit function" shows the $f_{\mathrm{n}}\left(B_{\mathrm{i}}\right)$ for each individual star. Red points show stars where $P / \Delta P>2.0$. The lower statistical probabilities of outlying polarization position angles for significant detections (e.g. $P / \Delta P>2.0$ ) is apparent by the non-compliant position angles in the rear-left corner of the figure. Insignificant polarizations would not be expected in this corner since their position angles will tend to be statistically random so that $\sin \left(\theta_{\mathrm{n}, \mathrm{i}}\right)>0$.

The distribution in Fig. 7 can be used to identify the set of nearby stars with polarization position angles that are consistent with $\mathrm{B}_{\mathrm{POL}}$. These stars include stars where measurement uncertainties are either small, $P / \Delta P>2.0$, or are large but with small values of $F_{\mathrm{II}}\left(\mathrm{B}_{\mathrm{i}}\right)$ and $P / \Delta P<<2.0$. Appendix $\mathrm{A}$ lists the identifications of the top third of the stars with polarization position angles that provide the best match to $\mathrm{B}_{\mathrm{POL}}$ and have $P / \Delta P>2.0$. These stars are located on the front right of Fig. 7. Stars with significant polarization position angles that do not comply with the direction of $\mathrm{B}_{\mathrm{POL}}$ are in the rear left part of the figure, and represent candidate polarizations for tracing an unrecognized component of the local ISMF (see \$4.6).

Fig. 7 shows that the minimization method used to select out the best-fitting ISMF direction (\$3), will be affected both by the compliant stars in the front right-hand corner of the figure, where the values of the merit function being minimized are small, and by the non-compliant stars in the rear left corner where the probability that the observed polarization position angle corresponds to the true polarization angle (given by $\mathrm{B}_{\mathrm{POL}}$ ) is negligible. The general implication of Fig. 7 is that a non-negligible fraction of the polarization position angles that are significant $(P / \Delta P>2)$ do not have polarization position angles that conform to (or are compliant with) the best-fitting ISMF. Those points are represented by low probabilities and large $\sin \left(\theta_{\mathrm{n}, \mathrm{i}}\right)$ values in the figure. Stars with small values of $F_{\mathrm{II}}\left(\mathrm{B}_{\mathrm{i}}\right)$ are refereed to as stars that 'conform' to, or are 'compliant' with, the dominant ISMF direction $\mathrm{B}_{\mathrm{POL}}$.

Fig. 8 shows the probability distribution of the stars as a function of the polarization position angle, $\theta_{\mathrm{POL}}$, evaluated with respect to the best-fitting ISMF, $\mathrm{B}_{\mathrm{POL}}$. Clearly stars

\footnotetext{
2 "North" refers to a geometric location and not the magnetic polarity in this context.
} 
with large measurement uncertainties are more likely to be compliant with the best-fitting ISMF $B_{\mathrm{POL}}$ than stars with small measurement uncertainties and polarization vectors pointing in the wrong direction. This feature allows data with all levels of accuracy to be useful in the fitting process. The second salient property of Fig. 8 is that there are numerous polarization position angles that have small mean errors and also clearly do not trace the same magnetic field direction as $\mathrm{B}_{\mathrm{POL}}$ and the IBEX Ribbon ISMF.

Fig. 9 maps the stars in Fig. 7 in the galactic coordinate system, and codes the symbol of the star as to whether or not the polarization position angle is compliant with $\mathrm{B}_{\mathrm{POL}}$. The star set is divided into two halves, based on the median value of $F_{\mathrm{II}}\left(\mathrm{B}_{\mathrm{i}}\right)$ of 3.54 . The best-matching half of the stars, where $F_{I I}\left(B_{i}\right)<3.54$, are plotted with solid symbols. The least-compliant half of the stars, $F_{\mathrm{II}}\left(\mathrm{B}_{\mathrm{i}}\right)>3.54$ are plotted with "X's" (also see Fig. 7). Stars where $P / \Delta P>2.0$ are plotted with red symbols, and stars with $P / \Delta P<2.0$ are plotted with black symbols. The compliant stars with $P / \Delta P>2.0$ have a tendency to be located between galactic longitudes of $0^{\circ}$ and $90^{\circ}$ in the northern hemisphere, and follow that trend until they wrap around $\ell$ at negative latitudes of $\sim-50^{\circ}$ in the southern galactic hemisphere near the BICEP2 region (\$5.6). The conforming polarization position angles tend to follow into two extended distributions, one located roughly between $\ell, b=90^{\circ}, 50^{\circ}$, and $60^{\circ},-60^{\circ}$, and the other extending roughly between $70^{\circ}, 65^{\circ}$ and $-10^{\circ},-65^{\circ}$. There is a slight tendency for the southern hemisphere conforming polarization position angles to be located in the region of the original nearby dust "patch" identified by Tinbergen (1982) that extended to negative galactic latitudes in the fourth galactic quadrant $\left(\ell>270^{\circ}\right)$ of the galaxy.

\subsection{Turbulence of best-fitting ISMF $\mathrm{B}_{\mathrm{POL}}$}

The turbulence in $\mathrm{B}_{\mathrm{POL}}$ can be evaluated from the dispersion of the polarization position angles calculated with respect to the direction of the best-fitting ISMF direction $\mathrm{B}_{\mathrm{POL}}$. The third of the data sample that consists of the stars with ISMF directions that provide the best fit to $\mathrm{B}_{\mathrm{POL}}$ contains 114 stars. Twenty-nine of those stars (listed in Appendix A) have significant polarizations with $P / \Delta P>2.0$. A rough estimate of magnetic turbulence can be found by evaluating the polarization position angles in a coordinate system that is aligned with the pole of $\mathrm{B}_{\mathrm{POL}}$. The average position angle for this 29-star subset is $\theta_{\mathrm{POL}}=12.0^{\circ} \pm 6.6^{\circ}$. This relatively small dispersion about the mean suggests that $\mathrm{B}_{\mathrm{POL}}$ has a low level of magnetic turbulence, and is not twisted by the kinematical properties of the CLIC (\$5.5).

A better estimate of magnetic turbulence is obtained by considering only 21st century

data that tend to have smaller mean errors than the older data. In the ideal case, the 
magnetic turbulence can be recovered by comparing the observed position angle variations with the mean measurement errors, or:

$$
\Phi_{\mathrm{IS}}^{2}=\operatorname{std}\left(\theta_{\mathrm{POL}}\right)^{2}-\operatorname{std}\left(\delta \theta_{\mathrm{me}}\right)^{2}
$$

The quantity $\Phi_{\text {IS }}$ represents the calculated interstellar turbulence (in degrees), $\operatorname{std}\left(\theta_{\mathrm{POL}}\right)$ is the standard deviation of the polarization position angle $\theta_{\mathrm{POL}}$ evaluated for a coordinate system with the pole located at $\mathrm{B}_{\mathrm{POL}}$, and $\operatorname{std}\left(\delta \theta_{\mathrm{me}}\right)$ is the standard deviation of the mean measurement errors of the data subset.

The amount of interstellar turbulence obtained from eqn. 4 varies with the number of stars that are included in the data subsample. If this subgroup of high-quality polarization data is sorted numerically according to goodness-of-fit between $\theta_{\mathrm{POL}}$ and $\mathrm{B}_{\mathrm{POL}}$, where the perfect measurement will have $\theta_{\mathrm{POL}}=0^{\circ}$, then the end-point of the array that contains the stars with the very best matches between the polarization position angles and $\mathrm{B}_{\mathrm{POL}}$ should also provide the best estimate of the interstellar magnetic turbulence using eqn. 4. In Fig. 10 we evaluate interstellar turbulence using eqn. 4 and by starting with the highest quality data set established by setting some minimum value for $P / \Delta P$. That data subset is then reevaluated by successively rejecting the lowest quality data points until a reasonable estimate for the interstellar turbulence is obtained. Fig. [10 shows the estimates of interstellar turbulence (solid lines) for two data subsets with $P / \Delta P>2.0$ (black lines) and $P / \Delta P>3.5$ (purple lines). The horizontal axis shows the number of qualifying stars included in the numbers used in eqn. 4, with the stars with polarization position angles that better match $\mathrm{B}_{\mathrm{POL}}$ on the figure right, and those with poorer matches on the figure left. For a data subset that is restricted to stars with $P / \Delta P>3.5$, the minimum value of the interstellar turbulence is $8^{\circ}$ and the seven stars that bracket this minimum have $\Phi_{\mathrm{IS}}=9^{\circ} \pm 1^{\circ}$.

For a larger subset where $P / \Delta P>2.0$, the minimum of the interstellar turbulence decreases to $\sim 2^{\circ}$ for the stars that best-comply with $\mathrm{B}_{\mathrm{POL}}$. However, the turbulent component of the interstellar magnetic field $\mathrm{B}_{\mathrm{POL}}$ should be best defined by the most precise data, so we report the turbulence $\Phi_{\mathrm{IS}}=9^{\circ} \pm 1^{\circ}$ from the $P / \Delta P>3.5$ data subset as the our best estimate of the turbulence of $\mathrm{B}_{\mathrm{POL}}$.

\subsection{Polarization data not assigned to an ISMF structure}

The large number of non-conforming stars in Fig. 9 suggests that one or more additional ISMF directions, not yet accounted for, must be influencing some of the polarization position angles. $\mathrm{B}_{\mathrm{POL}}$ is based on 343 measurements, of which $33 \%$ have $P / \Delta P>2.0$. The set of stars

with the largest third of the values of the $F_{\mathrm{II}}\left(\mathrm{B}_{\mathrm{i}}\right)$ (i.e. the one-third of the polarization data 
that are least compliant with the direction $\mathrm{B}_{\mathrm{POL}}$ ) were selected to be tested independently for a direction of the ISMF using the method described in $\$ 3$. The best-fitting ISMF direction for this third of the stars is toward $\ell=267.3^{\circ}, b=33.0^{\circ}$, which is $86^{\circ}$ from the heliosphere nose and is marginally constrained since it is at the edge of the region that is included in this study.

\section{Discussion}

\subsection{Comparing the magnetic field directions obtained from the polarization data and the IBEX ENA Ribbon}

IBEX measures ENAs created from charge-exchange between neutral interstellar atoms and heliosheath ions, including the solar wind and incorporated pickup ions (McComas et al. 2009; Livadiotis \& McComas 2012). IBEX discovered an extraordinarily circular Ribbon of ENAs that is about $20^{\circ}$ wide and several times more intense than the distributed flux of ENA emissions throughout the rest of the sky (McComas et al. 2009; Fuselier et al. 2009; Schwadron et al. 2011; Funsten et al. 2013). The locus of sightlines where the Ribbon is observed appear in directions where the ISMF draping over the heliosphere is perpendicular to the radial viewing sightline (Schwadron et al. 2009). There is no consensus agreement on the Ribbon formation mechanism (McComas et al. 2014). More recently, Schwadron \& McComas (2013) and Isenberg (2014) have suggested that the Ribbon is created through retention of pickup ions, implying that the Ribbon reflects a true spatial structure, not an optical effect due to the prominence of the pickup ring, as previously discussed (McComas et al. 2009; Heerikhuisen et al. 2010). The Ribbon geometry is a sensitive diagnostic of the ISMF direction and strength, and the pressure and ionization of the interstellar cloud surrounding the heliosphere (Frisch et al. 2010b; Heerikhuisen \& Pogorelov 2011; Ratkiewicz et al. 2012).

The IBEX Ribbon is a highly circular feature, with a radius of $74.5^{\circ} \pm 2.0^{\circ}$ that is centered on the ecliptic coordinates of $\lambda=219.2^{\circ} \pm 1.3^{\circ}, \beta=39.9^{\circ} \pm 2.3^{\circ}$ (based on the weighted mean average over the energy passbands, Funsten et al. 2013). The Ribbon center corresponds to galactic coordinates of $\ell=34.7^{\circ} \pm 4.4^{\circ}, b=56.6^{\circ} \pm 2.6^{\circ}$. The Ribbon center is energy dependent and shifts by $9.2^{\circ}$ across the five energy bands of IBEX-HI, from $\ell=34.7^{\circ}, b=55.5^{\circ}$ for the $0.7-1.7 \mathrm{keV}$ bands, to $\ell=20.1^{\circ}, b=60.7^{\circ}$ for the $4.3 \mathrm{keV}$ band, in galactic coordinates.

The observed center of the Ribbon arc is likely to be within $5^{\circ}$ of the true ISMF direction outside of the heliosphere. MHD simulations of the Ribbon formation by Heerikhuisen et al. 
(2014) show that the center of the Ribbon arc is offset from the direction of the ISMF far upstream of the heliosphere by $5^{\circ}$ for interstellar field strengths of $3 \mu \mathrm{G}$. Comparisons between the pressures of the inner heliosheath plasma and the thermal and ram pressure of the LIC give an ISMF of $\sim 3.3 \mu \mathrm{G}$. A similar value of $\sim 3.1 \mu \mathrm{G}$ is found from the magnetic distortion of the heliotail (Schwadron et al. 2011). Photoionization models of the LIC that include energy sinks and sources also predict $\mathrm{a} \sim 2.7 \mu \mathrm{G}$ magnetic field strength from the equipartition of energy (Slavin \& Frisch 2008).

The direction of the best-fitting ISMF that is obtained from the data set that omits the filament stars, $\mathrm{B}_{\mathrm{POL}}$, agrees remarkably well with the magnetic field direction that is obtained from the center of the IBEX Ribbon arc. The angular separation between the magnetic field direction that dominates the results obtained from the polarization data, $\mathrm{B}_{\mathrm{POL}}$, and the IBEX Ribbon field direction is $7.6^{\circ}\left(+14.9^{\circ},-7.6^{\circ}\right)$ (Table 1). The uncertainties become larger if the energy variation of the Ribbon center is included. The alignment of $\mathrm{B}_{\mathrm{POL}}$ and $\mathrm{B}_{\mathrm{IBEX}}$ indicate that these two magnetic field directions are the same to within the uncertainties, and that the interstellar magnetic field interacting with the heliosphere extends into the upwind interstellar regions with minimal distortion outside of the draping region. It is also possible that $\mathrm{B}_{\mathrm{POL}}$ agrees with $\mathrm{B}_{\mathrm{IBEX}}$ because $\mathrm{B}_{\mathrm{IBEX}}$ is the nearest coherent magnetic structure in the sky and therefore has the largest angular extent of all possible ordered fields.

Stars with polarization position angles that differ from $\mathrm{B}_{\mathrm{POL}}$, and therefore from $\mathrm{B}_{\mathrm{IBEX}}$, have slightly stronger polarization strengths than those that do not agree. However, there does not appear to be any difference between the distances of the two subsets of the data. The third of the polarization data that provides the worst match to $\mathrm{B}_{\mathrm{POL}}$ consists of 114 measurements that have a mean $P / \Delta P$ of $2.4 \pm 1.5$. The third of the polarization set that provides the best match to $\mathrm{B}_{\mathrm{POL}}$ has a mean $P / \Delta P$ of $1.5 \pm 1.1$. Both sets of stars have mean distances of approximately $25 \pm 10$ pc. The lower mean significance of the polarizations that provide the best match to $\mathrm{B}_{\mathrm{POL}}$, and therefore $\mathrm{B}_{\mathrm{IBEX}}$, is not surprising since total dust column densities will be lowest for the ISM closest to the heliosphere.

The polarity of the ISMF is not given by either the IBEX Ribbon data or by the polarization data. Both the polarity of the ISMF direction found by Voyager 1 at the heliopause (Burlaga \& Ness 2014), and the radio rotation measures of pulsars within several hundred parsecs in the fourth galactic quadrant (Salvati 2010), suggest a polarity for the local ISMF that is directed upwards through the galactic plane. 


\subsection{Galactic cosmic ray asymmetries}

Asymmetries in the flux of $\mathrm{TeV}$ galactic cosmic rays at Earth are observed over both large (Nagashima et al. 1998; Abdo et al. 2009) and small (Tibet As r Collaboration et al. 2011; Vernetto et al. 2009; Abbasi et al. 2011) angular scales. The cosmic rays in the 1.5-10 $\mathrm{TeV}$ energy range have gyroradii of $\sim 100-700 \mathrm{AU}$ in a $3 \mu \mathrm{G}$ magnetic field, and probe the magnetic field in the same spatial region as the IBEX Ribbon (Schwadron et al. 2014). Schwadron et al. modeled galactic cosmic ray streaming along the ISMF, for a small ratio of the perpendicular-to-parallel component of diffusion, and showed that the observed $\mathrm{TeV}$ cosmic ray asymmetries show a general ordering about the equator of $\mathrm{B}_{\mathrm{IBEX}}$ locally. Over larger spatial scales, interstellar magnetic turbulence may disrupt GCR streaming and reduce the magnitude of the GCR asymmetries.

The low level of magnetic turbulence found for $\mathrm{B}_{\mathrm{POL}}, \Phi_{\mathrm{IS}} \sim 9^{\circ} \pm 1^{\circ}$, indicates that the IBEX magnetic field extends out into interstellar space where the low magnetic turbulence does not impede the flux of TeV GCRs into the heliosphere. Over spatial scales of several

hundred parsecs, Salvati (2010) used the rotation measures of radio sources to determine a direction for the ISMF in the third galactic quadrant that is within $\sim 22^{\circ}-24^{\circ}$ of $\mathrm{B}_{\mathrm{IBEX}}$. Both the polarization data and the radio rotation measure data suggest that the IBEX Ribbon traces a non-turbulent magnetic field that extends into the third galactic quadrant from whence the GCR streaming arrives.

\subsection{Possible origins of the magnetic filament}

The origin of the filamentary structure (or structures) defined by the polarization position angles plotted in Fig. 6 is not firmly established. The filament polarizations trace an ISMF direction that is aligned with the direction of the heliosphere nose, and the filament stars are spatially arranged along a direction that is perpendicular to the

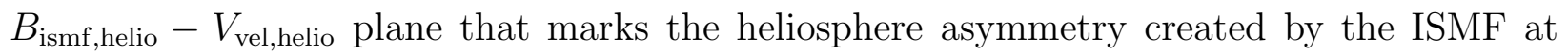
the heliosphere, $B_{\text {ismf,helio }}$ and the heliocentric interstellar gas velocity at the heliosphere, $V_{\text {vel,helio }}$. These properties led to the proposal that the filament polarizations are evidence for the deflection of the polarizing interstellar dust grains around the heliosphere (Frisch et al. 2015a). Confirmation of a filament origin in the outer heliosheath will require modeling the alignment and transport of interstellar dust grains during their approach to, and interaction with, the heliosphere. As this modeling is not yet available, other possible origins for the filament are briefly mentioned.

An alternate origin for the filament polarizing grains could be that the grains are near 
the Sun and embedded in the LIC flow, but outside the influence of the heliosphere. Since the ISMF that is the best fit to the filament polarizations coincides with the heliosphere nose direction, which is defined by the inflowing interstellar $\mathrm{He}^{\circ}$, this interpretation requires that either the ISMF traced by the filament is parallel to the heliocentric LIC gas velocity, or that the assumption of polarization vectors parallel to the magnetic field direction is invalid. The first requirement invokes a random coincidence between the filament ISMF direction and the LIC heliocentric velocity and violates the result that the LIC magnetic field and LSR velocity are perpendicular based on IBEX data (Table 1, Schwadron 2015). The second requirement may be fulfilled if radiative alignment is significant (e.g. Hoang \& Lazarian 2014; Andersson 2015).

Alternatively, if $B_{\text {FIL }}$ is not associated with the LIC but is located at a distance of $\sim 5 \mathrm{pc}$ from the Sun, then the filament extent is about $1.7 \times 7.1 \mathrm{pc}$ in the plane of the sky. If this feature has the same density as the LIC $\left(n \sim 0.26 \mathrm{H}^{\circ}\right.$ nucleii $\mathrm{cm}^{-3}$, Model 26 in Slavin \& Frisch 2008), then the column density associated with the feature would be $\log \mathrm{N}\left(\mathrm{H}^{\circ}\right)=18.14 \mathrm{~cm}^{-2}$. Such a column density would be consistent with other column densities through the very local ISM (Wood et al. 2005). However, the coincidence between the filament ISMF direction and the heliosphere nose would remain puzzling.

The filament could be associated with an unidentified magnetic field component, perhaps associated with a shock front related to Loop I that extends very close to the Sun. For this possibility, again, it is a coincidence that the best-fitting ISMF to the filament polarizations is toward the heliosphere nose. The shock could be associated with the ISM in front of one of the filament stars, $\alpha$ Oph (HD 159561, A5 III, 14 pc), where high abundances of refractory elements in the gas, a strong $\mathrm{Mg}^{\circ}$ line indicating high temperature or electron densities, and temperatures up to $23,000-60,000 \mathrm{~K}$ indicate the processing of dust through interstellar shocks (Frisch 1981; Frisch et al. 1987, 1999; Crawford 2001). The polarizations of the star HD 159561 that traces the filament was measured at high sensitivity by both PlanetPol (Bailey et al. 2010) and POLISH2 (Wiktorowicz et al. 2015), with good agreement between the polarization position angles.

Fig. 11 compares polarization directions with the configuration of dust reddening that is associated with the parts of Loop I within $\sim 100$ pc (Appendix B contains additional information about the figure). Some of the filament stars have polarizations that are loosely parallel to the edge of the cavity in the distribution of the dust extinction. The sample of polarized stars within $40 \mathrm{pc}$ has been divided into two, and the half with polarization position angles in best agreement with the very local ISMF $B_{\mathrm{FIL}}$ are circled in green. The filament polarizations for stars between $\ell=20^{\circ}$ and $\ell=90^{\circ}$ are not aligned with strong gradients in the cumulative color excess in Fig. 11, so a possible association between the 
filament and Loop I requires further study.

\subsection{Polarization efficiency in local ISM}

Measurements of starlight that has been polarized in the ISM provide one of the few viable methods for determining the distribution of nearby interstellar dust grains and understanding the relative distributions of interstellar gas and dust over parsec-sized scale lengths. Polarization efficiencies in the local ISM can be found by comparing polarization strengths with color excess $\mathrm{E}(\mathrm{B}-\mathrm{V})$, and $\mathrm{E}(\mathrm{B}-\mathrm{V})$ with hydrogen column densities. Column densities of $\mathrm{H}^{\circ}$ for stars within $40 \mathrm{pc}$ are typically less than $\mathrm{N}\left(\mathrm{H}^{\circ}\right) \leq 10^{18.7}$ (Wood et al. 2005). Using the mean ratio between $\mathrm{N}\left(\mathrm{H}^{\circ}\right)+2 \mathrm{NH}_{2}$ and $\mathrm{E}(\mathrm{B}-\mathrm{V})$ (Bohlin et al. 1978), and the upper envelope of the relation between polarization and E(B-V) (Serkowski et al. 1975) yields $\mathrm{E}(\mathrm{B}-\mathrm{V}) \leq 0.0009$ mag and $P_{\%} \leq 0.008$ for the very local ISM. Using instead the analogous relations that apply to low-extinction stars with low column densities of foreground $\mathrm{H}_{2}$ predicts $\mathrm{E}(\mathrm{B}-\mathrm{V}) \leq 0.001 \mathrm{mag}$ and $P_{\%} \leq 0.014$ in the local ISM. A quantitative discussion of the relations in this subsection that link hydrogen column density and polarization strength is given in Appendix C.

It might be expected that interstellar polarizations of nearby stars will be more effective than the polarizations of distant stars since the ISM toward distant sources is likely to be more complex, with different magnetic field directions or strong radiation fields in the sightline depolarizing a polarized beam. Variations in alignment efficiency are also indicated by theoretical calculations of perfectly aligned infinite cylinders that show approximately a factor 3-4 larger $P_{\%} / \mathrm{E}(\mathrm{B}-\mathrm{V})$ than is observed (Mathis 1979; Kim \& Martin 1994). The efficiency of nearby interstellar polarization can be tested using stars where both polarization data and hydrogen column density are available. Two variables are introduced to trace the efficiency of the polarization in a sightline, $\alpha$ and $\alpha$. The ratio $P_{\%} / \mathrm{E}(\mathrm{B}-\mathrm{V})$ is proportional to $\alpha$ and $\alpha$ for high-column density and low column density sightlines, respectively. Since $\mathrm{N}\left(\mathrm{H}^{\circ}\right)$ will be used as a proxy for $\mathrm{E}(\mathrm{B}-\mathrm{V})$ for the nearby stars (Appendix $\mathrm{C}$ ) a third variable $\gamma=\mathrm{N}\left(\mathrm{H}^{+}\right) / \mathrm{N}\left(\mathrm{H}^{\circ}\right)$ is included to account for the possible presence of ionized hydrogen.

Both polarization and UV aborption line data are available for the nearby star HD 34029 ( $\alpha$ Aur, Capella, 13 pc Wood et al. 2002). Combining UV and FUV data on Capella, and adopting a model for the cloud length, Wood et al. determined column densities of $\log \mathrm{N}\left(\mathrm{H}^{\circ}\right)=18.24 \pm 0.07 \mathrm{~cm}^{-2}$ and $\log \mathrm{N}\left(\mathrm{H}^{+}\right)=18.08 \pm 0.65$ for the LIC in this direction, corresponding to $\gamma \sim 0.69$ (with large uncertainties, \pm 1.47 ). The polarization of Capella is $P(\%)=0.024 \pm 0.009$ (Piirola 1977). Combining these values with eqns. D4 and D8 in Appendix $\triangle$ for reddened and unreddened sightlines respectively gives alpha $\sim 5.3$ and $\alpha \prime \sim 2.6$, 
where both estimates have large uncertainties. These large values for polarization efficiency compared to the expected values of one for the upper envelope of the relation between polarization and color excess of distant stars (Appendix C) suggest that polarization mechanisms in the local ISM are more efficient than for distant reddened and low-extinction stars. These results are nevertheless highly uncertain both because measurement uncertainties are large and Capella is a G1III+K0III binary system where intrinsic polarization is possible.

A second test of alignment efficiency in the local ISM is made using the two stars with spectral types not associated with intrinsic polarization, HD 11443 (F6IV, 20 pc) and 39587 (G0V, 9 pc) that have polarizations of $P_{\%}=0.02 \pm 0.009$ and $0.019 \pm 0.008$ respectively (Piirola 1977). For these stars $\log \mathrm{N}\left(\mathrm{H}^{\mathrm{o}}\right)=18.33 \mathrm{~cm}^{-2}$ and $\log \mathrm{N}\left(\mathrm{H}^{\mathrm{o}}\right)=17.93 \mathrm{~cm}^{-2}$, respectively (Wood et al. 2005). Information on $\mathrm{H}^{+}$toward these stars is not available so the Capella value for $\gamma$ is assumed. The resulting alignment efficiencies are $\alpha=3.6$, and $\alpha \prime=1.9$ for HD 11443, and $\alpha=8.5$ and $\alpha \prime=3.7$ for HD 39587. If lower ionization levels had been assumed, such as $\gamma \sim 0.29$ corresponding to the fractional ionization of the LIC near the heliosphere (Model 26 in Slavin \& Frisch 2008), these values of $\alpha$ and $\alpha \prime$ would increase.

These estimates of the polarization efficiency in the local ISM, where $\alpha>1$, are consistent with the results of Fosalba et al. (2002) who found a non-linear increase in polarization as extinction approached zero for low column density stars (see Appendix C). The low column density values of $\alpha \prime>1$ found in the previous paragraph could be spurious, resulting from the combination of the large uncertainties and this non-linear behavior of polarization strengths at low column densities. Further data on both interstellar column densities and polarizations toward the same stars are needed to establish the efficiency of the polarization mechanisms in the local ISM.

\subsection{Nearby magnetic field, Loop I superbubble, and the local interstellar cloud}

The agreement between $\mathrm{B}_{\mathrm{IBEX}}$ and $\mathrm{B}_{\mathrm{POL}}$ (Table 1) indicates that $\mathrm{B}_{\mathrm{IBEX}}$ extends into the interstellar medium without significant distortion. The low level of magnetic turbulence for $\mathrm{B}_{\mathrm{POL}}(\$ 4.5)$ suggests that the ratio of the plasma thermal to magnetic pressure, $\beta$, is $\leq 1$. A LIC magnetic field strength of $\sim 3 \mu \mathrm{G}$ is consistent with the total interstellar pressure required to balance the inner heliosheath plasma traced by IBEX ENAs, and the deflection 
of the heliotail to the port side 3 of the heliosphere (Schwadron et al. 2011), and also the equipartition of energy between the LIC thermal gas and magnetic field (Slavin \& Frisch 2008). Nearby polarized stars with significant polarizations that do not trace $\mathrm{B}_{\mathrm{POL}}(\$ 4.6)$ may indicate local regions where the magnetic field has been distorted by cloud motions that create high magnetic pressure (Fig. 13), or by cloud collisions (Fig. 12).

The local magnetic field, $\mathrm{B}_{\mathrm{POL}}$, provides a probe of the physical properties and origin of the interstellar cloudy material in the immediate solar neighborhood. The bulk motion through space of the nearby interstellar material associated with the CLIC, $<30 \mathrm{pc}$, has been determined from the velocities for interstellar optical and ultraviolet absorption lines and the flow of interstellar dust through the heliosphere (Frisch et al. 2011). The angle between $\mathrm{B}_{\mathrm{POL}}$ and the upwind direction of the CLIC LSR velocity ( $\mathrm{V}_{\mathrm{CLIC,LSR}}$, Appendix D) is $76.8^{\circ}\left(+23.5^{\circ},-27.6^{\circ}\right)$. Although the uncertainties are large, the bulk motion of local interstellar gas relative to the LSR is therefore perpendicular to the ISMF direction. The flow of the CLIC relative to the LSR originates in a direction that is within $21.4^{\circ} \pm 20.8^{\circ}$ of the nominal center of the Loop I superbubble. For the Loop I bubble center, we use the S1 shell feature, centered at $\ell=346^{\circ} \pm 5^{\circ}, b=3^{\circ} \pm 5^{\circ}$ as defined by (Wolleben 2007). The S1 bubble model places the Sun in the rim of the $\mathrm{S} 1$ shell. The relative configurations of $\mathrm{B}_{\mathrm{POL}}$, $\mathrm{V}_{\text {CLIC,LSR }}$, and the S1 bubble form a self-consistent picture where local ISM, consisting of the CLIC, is part of the rim of the Loop I superbubble and $B_{P O L}$ represents the magnetic field swept up in the rim of the expanding superbubble.

The IBEX measurements of the LIC velocity and LIC magnetic field provide a precise set of data for comparisons between the LIC interstellar gas velocity and magnetic field vectors. The heliocentric velocity determined by IBEX for interstellar $\mathrm{He}^{\circ}$ flowing through the heliosphere corresponds to a LIC velocity with respect to the LSR of $\mathrm{V}_{\text {IBEX.LSR }}=17.2 \pm 1.9$ $\mathrm{km} \mathrm{s}^{-1}$ toward $\ell, b=141.1^{\circ} \pm 5.9^{\circ}, 2.4^{\circ} \pm 4.2^{\circ}$ (based on the velocity in Schwadron et al. 2015). The direction of the ISMF that shapes the heliosphere is given by the weighted mean center of the IBEX Ribbon, at $\ell=34.8^{\circ} \pm 4.3^{\circ}, b=56.6^{\circ} \pm 1.2^{\circ}$ (Table 11, Funsten et al. 2013). The perpendicular angle between the LIC velocity with respect to the LSR, and the IBEX magnetic field direction $\left(96.9^{\circ} \pm 8.5^{\circ}\right.$, also see Schwadron et al. 2014) indicates that the motion of the LIC through space is consistent with a scenario where the partially ionized LIC (Model 26 in Slavin \& Frisch 2008, indicates that $\sim 22 \%$ of the hydrogen and $\sim 39 \%$ of

\footnotetext{
3The nautical terms "starboard" and "port" have been adopted to describe the flanks of the heliosphere to the right and left, respectively, of the heliosphere nose when referenced to the ecliptic coordinate system. In galactic coordinates this therefore places the starboard side of the heliosphere mostly in the fourth galactic quadrant at positive latitudes, and the port side of the heliosphere in the fourth galactic quadrant and mostly at negative latitudes.
} 
the helium are ionized) sweeps up and carries a frozen-in magnetic field through space.

Different aspects of this picture emerge when the 15-cloud model of Redfield \& Linsky (2008) is compared with the direction of the interstellar field. The angles between the LSR velocities of the fifteen clouds (given in Frisch \& Schwadron 2014) and B IBEX are plotted in Fig. 12 against $V_{\mathrm{LSR}}$. The magnetic field direction is represented by the IBEX value, as $\mathrm{B}_{\text {IBEX }}$ is known more precisely than the field determined from the polarization data, $\mathrm{B}_{\mathrm{POL}}$, and the two field directions agree (Table 1). A prominant characteristic of Fig. 12 is that, except for the Aur cloud, the LSR velocities of these clouds tend to increase as the angle between $V_{\mathrm{LSR}}$ and the interstellar field increases if the uncertainties are included. The mean angle between the $15 \mathrm{LSR}$ velocities and $\mathrm{B}_{\mathrm{IBEX}}$ is $107.5^{\circ}$.

If we assume that deviations of the velocities of the 15 individual clouds from the bulk CLIC motion are caused by the injection of energy into the CLIC gas, and that the energy injection is ordered by the pole of the IBEX ISMF direction $\mathrm{B}_{\mathrm{POL}}$ (Table 1), then the most effective acceleration is directed toward the antipode of $\mathrm{B}_{\mathrm{POL}}$ at $\ell=216^{\circ}, b=-49^{\circ}$. The largest deviations from the perpendicularity of the gas and magnetic field therefore occurs for higher velocities that are more pointed toward the third galactic quadrant, where gas and dust densities are extremely low (e.g. Fitzgerald 1968; Frisch \& York 1983; Vergely et al. 2010).

The CLIC is a decelerating flow of interstellar gas so that cloud collisions supply an opportunity for shock formation (Frisch et al. 2002; Gry \& Jenkins 2014; Redfield \& Linsky 2008; Linsky et al. 2008; Frisch et al. 2015c). Stars that are compliant with $\mathrm{B}_{\mathrm{POL}}$ are preferentially located in the first galactic quadrant, $\ell=0^{\circ}-90^{\circ}$ (Fig. 13). Some of the stars that have polarizations that are compliant with $\mathrm{B}_{\mathrm{POL}}(\$ 4.4$. Fig. 9) are also located in kinematically active regions. The elongated feature of compliant stars with $\ell=60^{\circ}-90^{\circ}$ (\$4.4) occupies a kinematically quiescent region (Fig. 13) dominated by the LIC, while the second elongated feature with $\ell=-10$ to $70^{\circ}$ occurs in a kinematically active region (Fig. 13) where multiple clouds and large differences in absorption component velocities in the same sightline indicate cloud collisions at up to $50 \mathrm{~km} \mathrm{~s}^{-1}$ (Linsky et al. 2008; Redfield \& Linsky 2008)

It is notable that there is minimal nearby polarization associated with the G-cloud centered near $\ell, b=315^{\circ}, 0^{\circ}$ (Fig. 13, Fig. 14). Two stars with polarization position angles that comply with $\mathrm{B}_{\mathrm{POL}}$ are found toward the G-cloud (Fig. 13). Two layers of nearby polarizing dust are found in the direction of the G-cloud, at $\sim 19$ pc and $\sim 55-65$ pc (Fig. 14). The G-cloud extends in front of $\alpha$ Cen, at $1.3 \mathrm{pc}$, but the polarizing grains are much more distant. 
The G-cloud region shows evidence of dust grain destruction by interstellar shocks. The average volume densities of interstellar $\mathrm{Fe}$ and $\mathrm{Ca}$ are systematically larger in the third and fourth galactic quadrants where the G-cloud is located, compared to $\ell<180^{\circ}$ (Frisch 2010 ). Destruction of dust grains in interstellar shocks preferentially returns refractory elements such as Fe and Ca to the gas phase (Jones et al. 1994; Frisch et al. 1999), so that the higher $\mathrm{Fe}$ and $\mathrm{Ca}$ densities in the G-cloud region may indicate recent shock activity and magnetic turbulence.

\subsection{Starlight polarizations in the BICEP2 field}

Interstellar polarization data provide one technique for selecting the dust-free sightlines that provide the optimum conditions for measurements of the B-mode polarization of the cosmic microwave background. BICEP2 has studied the B-mode polarization in a southern region centered near $\mathrm{RA}=0^{\circ}, \mathrm{DEC}=-57.5^{\circ}$ (Ade \& the BICEP2 team 2014). The BICEP2 field coincides roughly to the region defined by declinations between $-68^{\circ}$ and $-48^{\circ}$, and right ascensions between $322^{\circ}$ and $38^{\circ}$. This field, centered on galactic coordinates $\ell, b=$ $316.1^{\circ},-58.3^{\circ}$, is outlined with dotted lines on a smoothed map of color excess $\mathrm{E}(\mathrm{B}-\mathrm{V})$ for stars within 100 pc in Fig. 11 (also see Fig. 3). Fig. 15 shows that the BICEP2 region contains significant polarization within $40 \mathrm{pc}$ of the Sun, and with increasing polarization strengths out to $300 \mathrm{pc}$. The star that is circled at the high-longitude end of the BICEP2 region (Fig. 11) indicates a star with a polarization position angle that conforms to $\mathrm{B}_{\mathrm{POL}}$, and therefore to $\mathrm{B}_{\text {IBEX }}$, which suggests that $\mathrm{B}_{\mathrm{IBEX}}$ extends up to the edge of the BICEP2 region. A slightly different ISMF direction is traced by most of polarized stars in the BICEP2 region.

We have tested the dust content of the BICEP2 field using two markers of interstellar dust, optically polarized starlight and the color excess $\mathrm{E}(\mathrm{B}-\mathrm{V})$ of stars. The polarization data for stars in this field are plotted against distance in Fig. 15, using the data sources mentioned in \$2. Both significant polarization detections where $P / \Delta P \geq 2.0$, and lower polarization strengths that are not statistically significant are found. Polarizations are up to $\sim 0.2 \%$, corresponding to color excess values of $\mathrm{E}(\mathrm{B}-\mathrm{V}) \geq 0.028 \mathrm{mag}$ (using the low extinction relations of eqn. C5 for $\left.\alpha^{\prime} \leq 1\right)$, optical extinction $A_{\mathrm{V}}=3.1 * \mathrm{E}(\mathrm{B}-\mathrm{V}) \geq 0.087 \mathrm{mag}$ for an assumed selective-to-total extinction of 3.1, and column densities of $N(\mathrm{H}) \geq 1.4 \times 10^{20}$ $\mathrm{cm}^{-2}$. The starlight reddening data that give the color excess values in Fig. 11 (see Appendix B) indicate a larger color excess of $\mathrm{E}(\mathrm{B}-\mathrm{V})=0.19 \mathrm{mag}$ through the BICEP2 field, or optical extinction $A_{\mathrm{V}}=0.59 \mathrm{mag}$ for a selective-to-total extinction ratio of 3.1 .

Although the extinction of the BICEP2 region is negligible when compared to molecular 
clouds, the polaraization data show that the dust that is present produces detectable amounts of optical polarization in the starlight. Both $\mathrm{B}_{\mathrm{POL}}$ and the lower latitudes of Loop I extend into the BICEP2 region where they indicate that the local contributions to polarizations are likely to trace an ordered magnetic field with directions that can be predicted from the measurements of the very local ISMF $\mathrm{B}_{\mathrm{POL}}$ and the southerly portion of Loop I.

\section{Conclusions}

Starlight that is linearly polarized while traversing the dichroic local ISM is utilized to chart the direction of the magnetic field within $40 \mathrm{pc}$ and $90^{\circ}$ of the nose of the heliosphere, corresponding roughly to the galactic center hemisphere. The purpose of the study is to compare the direction of the ISMF that shapes the heliosphere, and is traced by the unconventional magnetic field diagnostic provided by the IBEX ENA Ribbon, with the local ISMF direction traced by the polarization data.

New data on polarized starlight acquired in the northern and southern hemispheres provide the basis for this study. These polarization measurements, with typical $3 \sigma$ sensitivities of $0.01 \%$, have been collected with telescopes at the KVA, LNA, and Lick observatories. Using the new polarization data and additional data from Paper II and the literature, we determine several nearby magnetic structures, one of which coincides in direction with the interstellar magnetic field traced by the IBEX Ribbon. Summarizing the results:

- A merit function, $F_{\mathrm{II}}\left(\mathrm{B}_{\mathrm{i}}\right)$, has been developed for evaluating the magnetic field direction that best fits the polarization data. $F_{\mathrm{II}}\left(\mathrm{B}_{\mathrm{i}}\right)$ assumes that the linear interstellar polarizations are parallel to the magnetic field direction. Evaluation of the merit function for the entire qualifying set of polarization data, within $40 \mathrm{pc}$ and $90^{\circ}$ of the heliosphere nose direction, results in a local ISMF direction in agreement with earlier values in Paper II.

- A visually and numerically distinct magnetic filament is traced by the polarizations of thirteen stars, of which the nearest star is within six parsecs. These filament stars were originally selected based on a gradient of the polarization position angles with distance, for position angles given in the galactic coordinate system, but that effect was found to be spurious $(\$ 4.2)$. Utilizing the function $F_{\mathrm{II}}\left(\mathrm{B}_{\mathrm{i}}\right)$ to obtain the ISMF direction that is traced by the filament polarizations gives an ISMF direction that is located within $15^{\circ} \pm 10.3^{\circ}$ of the heliosphere nose. Frisch et al. (2015a) favor an origin for the filament polarizations related to the interstellar dust that is deflected around the heliosphere 
in the outer heliosheath. Other possibilities are that the feature is associated with the local ISM or possibly related to the near side of Loop I.

- The ISMF that shapes the heliosphere, $\mathrm{B}_{\mathrm{IBEX}}$, has been identified in linearly polarized starlight for the first time. When the filament stars are omitted from the polarization data set that is evaluated with the merit function $F_{\mathrm{II}}\left(\mathrm{B}_{\mathrm{i}}\right)$, the ISMF direction of $\mathrm{B}_{\mathrm{POL}}$ is aligned with the direction $\ell=36.2^{\circ}, b=49.0^{\circ}\left( \pm 16^{\circ}\right)$. The direction of $\mathrm{B}_{\mathrm{POL}}$ is centered $7.6^{\circ}\left(+14.9^{\circ},-7.6^{\circ}\right)$ away from the ISMF direction found from the IBEX Ribbon (Table 1). The polarization position angles of the stars that best comply with $\mathrm{B}_{\mathrm{POL}}$ indicate that the magnetic turbulence of $\mathrm{B}_{\mathrm{POL}}$ is weak (\$4.5). Several of the stars that trace $\mathrm{B}_{\mathrm{POL}}$ are located within $\sim 10 \mathrm{pc}$. $\mathrm{B}_{\mathrm{POL}}$ is not distinguished by the mean distance of the stars that trace $\mathrm{B}_{\mathrm{POL}}$, but those stars tend to show weaker polarizations than average.

- The local ISMF must be complex because many stars have polarization position angles that trace a magnetic field direction that is different from $\mathrm{B}_{\mathrm{POL}}$.

- $\mathrm{B}_{\mathrm{POL}}$ must thread the local interstellar clouds. It forms an angle of $76.8^{\circ}\left(+23.5^{\circ},-27.6^{\circ}\right)$ with the bulk CLIC motion relative to the LSR. This result agrees with previous findings and is consistent with a model where the CLIC is associated with an evolved rim of the Loop I superbubble.

- The velocities of the 15 CLIC clouds identified by Redfield \& Linskv (2008) have been converted into the LSR; those LSR velocities increase as the angle between the LSR velocity and $\mathrm{B}_{\mathrm{POL}}$ increases. This suggests that $\mathrm{B}_{\mathrm{POL}}$ orders the kinematics of the local ISM. One possible scenario is that the polarized dust bridge extending from the heliosphere to the North Polar Spur region represents dust and the magnetic fields swept up by the large-scale expansion of the Loop I superbubble.

- The polarizations of three stars within 20 pc have been compared with hydrogen column densities. Values of $\mathrm{E}(\mathrm{B}-\mathrm{V})$ were estimated from $\mathrm{N}\left(\mathrm{H}^{\circ}\right)$ and then compared with polarization strengths $(\$ 5.4)$. The resulting ratios $P_{\%} / \mathrm{E}(\mathrm{B}-\mathrm{V})$ are consisent with the results of Fosalba et al. (2002) that find an upturn in polarization strengths for lowextinction stars, although uncertainties are large.

- The polarization data indicate that $\mathrm{B}_{\mathrm{POL}}$, which coincides with $\mathrm{B}_{\mathrm{IBEX}}$, extends to the edge of the region that was tested by BICEP2 for the B-mode polarization of the CMB. Polarizations of up to $0.2 \%$ inside of the BICEP2 field are found, corresponding to a color excess of $\mathrm{E}(\mathrm{B}-\mathrm{V}) \geq 0.028 \mathrm{mag}$. Data on starlight reddening give a larger color excess, $\mathrm{E}(\mathrm{B}-\mathrm{V})=0.19 \mathrm{mag}$ in the BICEP2 field, corresponding to an optical extinction $A_{\mathrm{V}}=0.59 \mathrm{mag}$. 
The only nearby interstellar cloud where the relation between the gas and dust is clearly established is the LIC, where multiple spacecraft have measured UV LIC absorption lines and the ISMF direction has been found from heliosphere models and the IBEX Ribbon. Achieving a similar understanding of the relation between the local ISMF traced by the polarization data and interstellar clouds will require UV studies of the interstellar absorption lines toward the same stars for which high-quality polarization data are available. Only then will a full understanding of the relation between the ISMF that shapes the heliosphere and the magnetic field that is associated with interstellar clouds in the solar vicinity be possible.

This research has been partly supported by the NASA Explorer program through support for the IBEX mission, and by the European Research Council Advanced Grant HotMol (ERC-2011-AdG 291659). P. Frisch would like to thank Stephen Case for pointing out the first study of the solar apex motion in Herschel (1783).

\section{A. Appendix: Stars with polarizations that best conform to $\mathrm{B}_{\mathrm{POL}}$}

The 29 stars with $P / \Delta P \geq 2.0$ that belong to the third of the sample with polarization position angles that best match $\mathrm{B}_{\mathrm{POL}}$ (see \$4.4) are: HD 11276, HD 90132, HD 90355, HD 91324, HD 112413, HD 117939, HD 126660, HD 127762, HD 130109, HD 150680, HD 161892, HD 169916, HD 173818, HD 177409, HD 177716, HD 184509, HD 185395, HD 187642, HD 190248, HD 197989, HD 198149, HD 205478, HD 207129, HD 210027, HD 210049, HD 210418, HD 215696, HD 216435, HD 223889.

\section{B. Appendix: Color excess E(B-V)}

The color excess E(B-V) contours in Fig. 11 are based on the photometric and astromet-

ric data for stars brighter than $V=9$ mag in the Hipparcos catalog (Perryman 1997). Color excess values $\mathrm{E}(\mathrm{B}-\mathrm{V})$ are calculated using the intrinsic stellar colors as a function of spectral type given by Cox (2000). Poorly defined spectral types are weeded out by only using stars where astrometric distances match photometric distances to within 15\%. The uncertainties on the astrometric distances are used to spatially smooth the $\mathrm{E}(\mathrm{B}-\mathrm{V})$ values over $\pm 13^{\circ}$ angles in the sky for stars with astrometric distances that overlap. Variable stars are not included in the construction of the $\mathrm{E}(\mathrm{B}-\mathrm{V})$ maps. Variability is filtered out by excluding Hipparcos data with variabilities that are larger than $0.06 \mathrm{mag}$. The contour levels of $\mathrm{E}(\mathrm{B}-\mathrm{V})=0.01$, $0.04,0.09$, and $0.17 \mathrm{mag}$ correspond to foreground hydrogen column densities of 19.76, 20.37, 20.72 , and $20.99 \mathrm{~cm}^{-2}$ for $\mathrm{N}\left(\mathrm{H}^{o}+2 \mathrm{H}_{2}\right) / \mathrm{E}(\mathrm{B}-\mathrm{V})=5.8 \times 10^{21} \mathrm{~cm}^{-2} \mathrm{mag}^{-1}$ (Bohlin et al. 1978) 
providing that negligible amounts of $\mathrm{H}^{+}$are present.

\section{Appendix: Column densities, color excess and polarization strengths}

This appendix summarizes the relations between hydrogen column densities and polarization strengths as determined from the literature. The discussion in $\$ 5.4$ uses these relations to evaluate whether the polarizing mechanisms in the local ISM are more efficient than in the generic ISM.

The relations between color excess $\mathrm{E}(\mathrm{B}-\mathrm{V})$ and polarization strengths has been determined both for reddened stars (Serkowski et al. 1975) and lightly reddened stars (Fosalba et al. 2002). The upper envelope of the plot of color excess $E(B-V)$ vs.polarization strength for generic reddened stars within several kpc of the Sun is given by $P_{\%}=9 \mathrm{E}(\mathrm{B}-\mathrm{V})$, where $P_{\%}$ is percent polarization (Serkowski et al. 1975, §2). The upper envelope of the relation between polarization and color excess is found to be non-linear for lightly reddened stars, $\mathrm{E}(\mathrm{B}-\mathrm{V})<1$ mag, giving the alternate relation $P_{\%}^{\prime}=3.5 \mathrm{E}(\mathrm{B}-\mathrm{V})^{0.8}$ (eqn. 3 in Fosalba et al. 2002). The term "upper envelope" indicates the maximum polarization that has been observed as a function of extinction, and therefore by common assumption the maximum allowable polarization for a given level of extinction.

Obtaining the dependence of polarization on hydrogen column densities requires the use of an additional relation between extinction and column density. The Copernicus mission studied the absorption lines of nearby stars in the far- $\mathrm{UV}$ where the $\mathrm{H}^{\circ}$ Lyman lines and $\mathrm{H}_{2}$ absorption lines are located (Savage et al. 1977; Bohlin et al. 1978). Observations of $\mathrm{H}^{\circ}$ and $\mathrm{H}_{2}$ yielded a mean ratio of total neutral hydrogen to color excess of $\left\langle N\left(\mathrm{H}^{\circ}+\mathrm{H}_{2}\right)\right\rangle / \mathrm{E}(\mathrm{B}-$ $\mathrm{V})=5.8 \times 10^{21} \mathrm{~cm}^{-2} \mathrm{mag}^{-1}$. Copernicus did not directly measure column densities of ionized hydrogen, $\mathrm{N}\left(\mathrm{H}^{+}\right)$. The ionization corrections needed to obtain total hydrogen column densities obtained from $\mathrm{H}^{\circ}+\mathrm{H}_{2}$ are expected to be less then a few percent for the Copernicus

stars (Bohlin et al. 1978), nearly all of which are beyond $100 \mathrm{pc}$ and therefore beyond the boundaries of the Local Bubble that is nearly devoid of interstellar dust (Fitzgerald 1968).

Observed polarizations may differ from the relation expected from the upper envelope of the relation between polarization and extinction. We introduce the terms $\alpha$ and $\alpha$ ' to evaluate the polarization efficiency of reddened and unreddened sightlines, respectively. Variations in the terms $\alpha$ and $\alpha \prime$ can result from the presence of several different magnetic field directions foreground to the star, patchy dust distributions, and/or variations in the grain characteristics.

The total hydrogen column density is used as a proxy for extinction because the extinc- 
tion toward nearby stars is too low to be determined with the standard evaluation of the difference between the attenuation of light in the $\mathrm{B}$ and $\mathrm{V}$ passbands that creates the color excess $\mathrm{E}(\mathrm{B}-\mathrm{V})$. Hence a second factor that will influence the evaluation of the efficiency of alignment mechanisms is the fractional ionization of hydrogen. Ionization corrections are implemented through the term $\gamma=N\left(\mathrm{H}^{+}\right) / N\left(\mathrm{H}^{\circ}\right)$, where $\mathrm{N}\left(\mathrm{H}^{+}\right)$is the column density of ionized hydrogen.

Significant amounts of $\mathrm{H}_{2}$ are not expected in the local ISM because of high fluxes of far-UV and extreme-UV ionizing radiation (Vallerga 1998; Vallerga \& Slavin 1998) and low column densities(Wood et al. 2005). Locally the high EUV fluxes also generate significant amounts of $\mathrm{H}^{+}$in the LIC (Slavin \& Frisch 2008) and other clouds (Slavin \& Frisch 1998; Redfield \& Falcon 2008). The term $\mathrm{N}\left(\mathrm{H}^{\circ}\right)+2 \mathrm{~N}\left(\mathrm{H}_{2}\right)$ in (Bohlin et al. 1978) can therefore be replaced by $\mathrm{N}\left(\mathrm{H}^{\circ}\right)+\mathrm{N}\left(\mathrm{H}^{+}\right)=\mathrm{N}\left(\mathrm{H}^{\circ}\right)(1+\gamma)$ for the very local ISM.

For reddened stars and the mean $N(\mathrm{H}) / \mathrm{E}(\mathrm{B}-\mathrm{V})$ Copernicus relation:

$$
\begin{array}{r}
\mathrm{P}_{\%}=\alpha 9 \mathrm{E}(\mathrm{B}-\mathrm{V}) \\
C=1.55 \times 10^{-21} \mathrm{mag} \mathrm{cm}{ }^{2} \\
\mathrm{P}_{\%}=\alpha C\left(N\left(\mathrm{H}^{\circ}\right)+N\left(\mathrm{H}^{+}\right)\right) \\
\alpha=\frac{\mathrm{P}_{\%}}{C N\left(\mathrm{H}^{\circ}\right)(1+\gamma)}
\end{array}
$$

Low extinction stars have a slightly different ratio of hydrogen column density to E(BV). Restricting the Copernicus data sample to those stars with a small fraction of $\mathrm{H}_{2},<1 \%$, provided a sample of the "intercloud medium" where the mean ratio between $\mathrm{H}$ and $\mathrm{E}(\mathrm{B}$ $\mathrm{V})$ is $\left\langle N\left(\mathrm{H}^{\circ}+\mathrm{H}_{2}\right)\right\rangle / \mathrm{E}(\mathrm{B}-\mathrm{V})=5.0 \times 10^{21} \mathrm{~cm}^{-2} \mathrm{mag}^{-1}$ (Bohlin et al. 1978). For lightly reddened stars and the Copernicus relation for the intercloud medium the polarizationextinction measure becomes:

$$
\begin{array}{r}
\mathrm{P}_{\%}^{\prime}=\alpha^{\prime} 3.5 \mathrm{E}(\mathrm{B}-\mathrm{V})^{0.8} \\
C^{\prime}=1.53 \times 10^{-17} \mathrm{mag} \mathrm{cm}{ }^{2} \\
\mathrm{P}_{\%}=\alpha^{\prime} C^{\prime}\left(N\left(\mathrm{H}^{\circ}\right)+N\left(\mathrm{H}^{+}\right)^{0.8}\right) \\
\alpha^{\prime}=\frac{\mathrm{P}_{\%}}{C^{\prime} N\left(\mathrm{H}^{\circ}\right)^{0.8}(1+\gamma)^{0.8}}
\end{array}
$$

which can be used to estimate both $\alpha^{\prime}$ and $\gamma$ for sightlines where $\mathrm{H}^{\circ}$ and $\mathrm{H}^{+}$and polarization data are available, where $\alpha \prime$ is the efficiency of grain alignment in the absence of depolarization effects.

These relations are used to evaluate the polarization efficiency in the local ISM in $\$ 5.4$. 


\section{Appendix: Bulk motion of local interstellar clouds relative to the LSR}

Astrometric data collected by the founders of modern astronomy revealed that both the Sun and stars are moving through space (Herschel 1783), 4 and led to the recognition that the Sun encounters interstellar clouds during its journey through space (Shapley 1921). Because both the Sun and interstellar clouds move through space, the Doppler contribution of solar motion to the heliocentric interstellar velocities must be removed for comparisons between kinematically defined clouds and spatially defined objects such as Loop I.

For conversion to the LSR velocity frame, which traces the mean velocity of nearby gravitationally relaxed stars around the galactic center, we use the solar motion relative to the LSR derived by Schönrich et al. (2010). Using data from the Hipparcos spacecraft (Perryman 1997), the Schönrich et al. (2010) results for the U, V, and W solar velocity components correspond to a solar velocity of $\mathrm{V}=18.0 \pm 0.9 \mathrm{~km} \mathrm{~s}^{-1}$ toward $\ell=47.8^{\circ} \pm 2.9^{\circ}$, $B=23.8^{\circ} \pm 2.0^{\circ}$ (e.g. the solar apex motion). The direction determined by Herschel (1783), based on the nearest and brightest stars, was toward the star $\lambda$ Her that is located $5^{\circ}$ away from this direction.

The bulk motion through space of the nearby interstellar material associated with the CLIC, < 30 pc, been determined from the velocities for interstellar optical and ultraviolet absorption lines, as well as the flow of interstellar dust through the heliosphere. Observations of interstellar absorption lines in 96 stars sampling the nearby ISM have been used to determine the bulk flow of nearby ISM through space for the assumption that the material flows as a rigid-body (Frisch et al. 2002), yielding the heliocentric flow velocity of $28.1 \pm 4.6$ $\mathrm{km} \mathrm{s}^{-1}$ toward $\ell, b=192.4^{\circ},-11.6^{\circ}$. Uncertainties on the longitude or latitude were not originally provided, so for the purposes of evaluating the motion of the CLIC relative to the LSR, uncertainties of $2^{\circ}$ each are adopted for $\ell$ and $b$.

The heliocentric velocity of the CLIC, based on the bulk motion of the CLIC in Frisch et al. (2002), is given in Table D. The LIC LSR velocity in Table D is based on the IBEX $\mathrm{He}^{\circ}$ velocity in Schwadron et al. (2015).

\footnotetext{
4 "Now, if the proper motion of the stars in general be once admitted, who can refuse to allow that our sun, with all its planets and comets, that is, the solar sytsem, is no less liable to such a general agitation as we find to obtain among all the rest of the celestial bodies. Admitting this for granted, the greatest difficulty will be how to discern the proper motion of the sun between so many other (and variously compounded) motions of the stars. This is an arduous task indeed, which we must not hope to see accomplished in a little time; but we are not to be discouraged from the attempt. Let us, in all events, endeavour to lay a good foundation for those who are to come after us."
} 


\section{REFERENCES}

Abbasi, R., Abdou, Y., Abu-Zayyad, T., Adams, J., Aguilar, J. A., Ahlers, M., Altmann, D., Andeen, K., Auffenberg, J., Bai, X., \& et al. 2011, ApJ, 740, 16

Abdo, A. A., Allen, B. T., Aune, T., Berley, D., Casanova, S., Chen, C., Dingus, B. L., Ellsworth, R. W., Fleysher, L., Fleysher, R., Gonzalez, M. M., Goodman, J. A., Hoffman, C. M., Hopper, B., Hüntemeyer, P. H., Kolterman, B. E., Lansdell, C. P., Linnemann, J. T., McEnery, J. E., Mincer, A. I., Nemethy, P., Noyes, D., Pretz, J., Ryan, J. M., Parkinson, P. M. S., Shoup, A., Sinnis, G., Smith, A. J., Sullivan, G. W., Vasileiou, V., Walker, G. P., Williams, D. A., \& Yodh, G. B. 2009, ApJ, 698, 2121

Ade, P. A. R. \& the BICEP2 team. 2014, Physical Review Letters, 112, 241101

Andersson, B.-G. 2015, in Astrophysics and Space Science Library, Vol. 407, Astrophysics and Space Science Library, ed. A. Lazarian, E. M. de Gouveia Dal Pino, \& C. Melioli, 59

Andersson, B.-G. \& Potter, S. B. 2006, ApJ, 640, L51

Bailey, J., Kedziora-Chudczer, L., Cotton, D. V., Bott, K., Hough, J. H., \& Lucas, P. W. 2015, MNRAS, 449, 3064

Bailey, J., Lucas, P. W., \& Hough, J. H. 2010, MNRAS, 405, 2570

Berdyugin, A., Piirola, V., \& Teerikorpi, P. 2014, A\&A, 561, A24

Bohlin, R. C., Savage, B. D., \& Drake, J. F. 1978, ApJ, 224, 132

Burlaga, L. F. \& Ness, N. F. 2014, ApJ, 784, 146

Cox, A. N. 2000, Allen's Astrophysical Quantities (AIP Press), 29-30

Crawford, I. A. 2001, MNRAS, 327, 841

Fitzgerald, M. P. 1968, AJ, 73, 983

Fosalba, P., Lazarian, A., Prunet, S., \& Tauber, J. A. 2002, ApJ, 564, 762

Frisch, P. C. 1981, Nature, 293, 377

-. 1993, ApJ, 407, 198

-. 2010, ApJ, 714, 1679 
Frisch, P. C., Andersson, B., Berdyugin, A., Funsten, H. O., Magalhaes, A. M., McComas, D. J., Piirola, V., Schwadron, N. A., Seriacopi, D. B., Slavin, J. D., \& Wiktorowicz, S. J. 2015a, ApJ, 805, 60

Frisch, P. C., Andersson, B., Berdyugin, A., Funsten, H. O., Magalhaes, M., McComas, D. J., Piirola, V., Schwadron, N. A., Slavin, J. D., \& Wiktorowicz, S. J. 2010a, ApJ, 724,1473

Frisch, P. C., Andersson, B.-G., Berdyugin, A., Piirola, V., DeMajistre, R., Funsten, H. O., Magalhaes, A. M., Seriacopi, D. B., McComas, D. J., Schwadron, N. A., Slavin, J. D., \& Wiktorowicz, S. J. 2012, ApJ, 760, 106

Frisch, P. C., Berdyugin, A., Piirola, V., Funsten, H. O., Magalhaes, A. M., Seriacopi, D. B., McComas, D. J., Schwadron, N. A., \& Wiktorowicz, S. J. 2015b, Journal of Physics : 13th Annual International Astrophysics Conference: Voyager, IBEX and the Interstellar Medium., 577, ID: 012010

Frisch, P. C., Bzowski, M., Drews, C., Livadiotis, G., McComas, D. J., Moebius, E., Schwadron, N. A., \& Sokół, J. M. 2015c, ApJ, 801, 601

Frisch, P. C., Dorschner, J. M., Geiss, J., Greenberg, J. M., Grün, E., Landgraf, M., Hoppe, P., Jones, A. P., Krätschmer, W., Linde, T. J., Morfill, G. E., Reach, W., Slavin, J. D., Svestka, J., Witt, A. N., \& Zank, G. P. 1999, ApJ, 525, 492

Frisch, P. C., Grodnicki, L., \& Welty, D. E. 2002, ApJ, 574, 834

Frisch, P. C., Heerikhuisen, J., Pogorelov, N. V., DeMajistre, B., Crew, G. B., Funsten, H. O., Janzen, P., McComas, D. J., Moebius, E., Mueller, H., Reisenfeld, D. B., Schwadron, N. A., Slavin, J. D., \& Zank, G. P. 2010b, ApJ, 719, 1984

Frisch, P. C., Redfield, S., \& Slavin, J. 2011, ARA\&A, 49

Frisch, P. C. \& Schwadron, N. A. 2014, in Astronomical Society of the Pacific Conference Series, Vol. 484, Outstanding Problems in Heliophysics: From Coronal Heating to the Edge of the Heliosphere, ed. Q. Hu \& G. P. Zank, 42

Frisch, P. C. \& York, D. G. 1983, ApJ, 271, L59

Frisch, P. C., York, D. G., \& Fowler, J. R. 1987, ApJ, 320, 842

Funsten, H. O., Allegrini, F., Crew, G. B., DeMajistre, R., Frisch, P. C., Fuselier, S. A., Gruntman, M., Janzen, P., McComas, D. J., Möbius, E., Randol, B., Reisenfeld, D. B., Roelof, E. C., \& Schwadron, N. A. 2009, Science, 326, 964 
Funsten, H. O., DeMajistre, R., Frisch, P. C., Heerikhuijsen, J., Higdon, D. M., Janzen, P., Larsen, B., Livadiotis, G., McComas, D. J., Möbius, E., Reese, C., Reisenfeld, D. B., Schwadron, N. A., \& Zirnstein, E. J. 2013, ApJ, 776, 30

Fuselier, S. A., Allegrini, F., Funsten, H. O., Ghielmetti, A. G., Heirtzler, D., Kucharek, H., Lennartsson, O. W., McComas, D. J., Möbius, E., Moore, T. E., Petrinec, S. M., Saul, L. A., Scheer, J. A., Schwadron, N., \& Wurz, P. 2009, Science, 326, 962

Gry, C. \& Jenkins, E. B. 2014, A\&A, 567, A58

Heerikhuisen, J. \& Pogorelov, N. V. 2011, ApJ, 738, 29

Heerikhuisen, J., Pogorelov, N. V., Zank, G. P., Crew, G. B., Frisch, P. C., Funsten, H. O., Janzen, P. H., McComas, D. J., Reisenfeld, D. B., \& Schwadron, N. A. 2010, ApJ, 708, L126

Heerikhuisen, J., Zirnstein, E. J., Funsten, H. O., Pogorelov, N. V., \& Zank, G. P. 2014, ApJ, 784, 73

Heiles, C. 2000, AJ, 119, 923

Herschel, W. E. F. R. S. 1783, Phil. Trans. R. Soc. Lond., 73, 247

Hoang, T. \& Lazarian, A. 2014, MNRAS, 438, 680

Isenberg, P. A. 2014, ApJ, 787, 76

Jones, A. P., Tielens, A. G. G. M., Hollenbach, D. J., \& McKee, C. F. 1994, ApJ, 433, 797

Kim, S.-H. \& Martin, P. G. 1994, ApJ, 431, 783

Krueger, H., Strub, P., Sterken, V. J., \& Grün, E. 2014, submitted to ApJ

Landgraf, M., Baggaley, W. J., Grün, E., Krüger, H., \& Linkert, G. 2000, J. Geophys. Res., 105,10343

Linsky, J. L., Rickett, B. J., \& Redfield, S. 2008, ApJ, 675, 413

Livadiotis, G. \& McComas, D. J. 2012, ApJ, 749, 11

Mathis, J. S. 1979, ApJ, 232, 747 
McComas, D. J., Allegrini, F., Bochsler, P., Bzowski, M., Christian, E. R., Crew, G. B., DeMajistre, R., Fahr, H., Fichtner, H., Frisch, P. C., Funsten, H. O., Fuselier, S. A., Gloeckler, G., Gruntman, M., Heerikhuisen, J., Izmodenov, V., Janzen, P., Knappenberger, P., Krimigis, S., Kucharek, H., Lee, M., Livadiotis, G., Livi, S., MacDowall, R. J., Mitchell, D., Möbius, E., Moore, T., Pogorelov, N. V., Reisenfeld, D., Roelof, E., Saul, L., Schwadron, N. A., Valek, P. W., Vanderspek, R., Wurz, P., \& Zank, G. P. 2009, Science, 326, 959

McComas, D. J., Bzowski, M., Frisch, P. C., Fuselier, S. A., Kubiak, M. A., Kucharek, H., Leonard, T., Möbius, E., Schwadron, N. A., Sokol, J. M., Swaczyna, P., \& Witte, M. 2015, ApJ, 801, 28

McComas, D. J., Lewis, W. S., \& Schwadron, N. A. 2014, Reviews of Geophysics

Munch, G. \& Unsold, A. 1962, ApJ, 135, 711

Nagashima, K., Fujimoto, K., \& Jacklyn, R. M. 1998, J. Geophys. Res., 1031, 17429

Naghizadeh-Khouei, J. \& Clarke, D. 1993, A\&A, 274, 968

Pereyra, A. \& Magalhães, A. M. 2007, ApJ, 662, 1014

Perryman, M. A. C. 1997, A\&A, 323, L49

Piirola, V. 1977, A\&AS, 30, 213

Piirola, V., Berdyugin, A., \& Berdyugina, S. 2014, in Society of Photo-Optical Instrumentation Engineers (SPIE) Conference Series, Vol. 9147, Society of Photo-Optical Instrumentation Engineers (SPIE) Conference Series, 8

Ratkiewicz, R., Strumik, M., \& Grygorczuk, J. 2012, ApJ, 756, 3

Redfield, S. \& Falcon, R. E. 2008, ArXiv e-prints, 804

Redfield, S. \& Linsky, J. L. 2008, ApJ, 673, 283

Salvati, M. 2010, A\&A, 513, A28

Santos, F. P., Corradi, W., \& Reis, W. 2011, ApJ, 728, 104

Savage, B. D., Bohlin, R. C., Drake, J. F., \& Budich, W. 1977, ApJ, 216, 291

Schönrich, R., Binney, J., \& Dehnen, W. 2010, MNRAS, 403, 1829 
Schwadron, N. A., Adams, F. C., Christian, E. R., Desiati, P., Frisch, P., Funsten, H. O., Jokipii, J. R., McComas, D. J., Moebius, E., \& Zank, G. P. 2014, Science, 343, 988

Schwadron, N. A., Allegrini, F., Bzowski, M., Christian, E. R., Crew, G. B., Dayeh, M., DeMajistre, R., Frisch, P., Funsten, H. O., Fuselier, S. A., Goodrich, K., Gruntman, M., Janzen, P., Kucharek, H., Livadiotis, G., McComas, D. J., Moebius, E., Prested, C., Reisenfeld, D., Reno, M., Roelof, E., Siegel, J., \& Vanderspek, R. 2011, ApJ, 731, 56

Schwadron, N. A., Bzowski, M., Crew, G. B., Gruntman, M., Fahr, H., Fichtner, H., Frisch, P. C., Funsten, H. O., Fuselier, S., Heerikhuisen, J., Izmodenov, V., Kucharek, H., Lee, M., Livadiotis, G., McComas, D. J., Moebius, E., Moore, T., Mukherjee, J., Pogorelov, N. V., Prested, C., Reisenfeld, D., Roelof, E., \& Zank, G. P. 2009, Science, 326,966

Schwadron, N. A. \& McComas, D. J. 2013, ApJ, 764, 92

Schwadron, N. A., Moebius, E., Leonard, T., Fuselier, S. A., McComas, D. J., Heirtzler, D., Kucharek, H., Rahmanifard, F., Bzowski, M., Kubiak, M. A., Sokol, J., Swaczyna, P., \& Frisch, P. 2015, ApJS, in press

Schwadron, N. A. e. a. 2015, Astra

Serkowski, K., Mathewson, D. S., \& Ford, V. L. 1975, ApJ, 196, 261

Shapley, H. 1921, J. Geology, 29

Slavin, J. D. \& Frisch, P. C. 1998, in Berlin Springer Verlag Lecture Notes in Physics, The Local Bubble and Beyond, Vol. 506, 305-308

Slavin, J. D. \& Frisch, P. C. 2008, A\&A, 491, 53

Sterken, V. J., Strub, P., Krueger, H., von Steiger, R., \& Frisch, P. 2015, ApJ, in press, 00

Tibet As $\gamma$ Collaboration, Amenomori, M., Bi, X. J., Chen, D., Cui, S. W., Danzengluobu, Ding, L. K., Ding, X. H., Fan, C., Feng, C. F., Feng, Z., Feng, Z. Y., Gao, X. Y., Geng, Q. X., Guo, H. W., He, H. H., He, M., Hibino, K., Hotta, N., Hu, H., Hu, H. B., Huang, J., Huang, Q., Jia, H. Y., Kajino, F., Kasahara, K., Katayose, Y., Kato, C., Kawata, K., Labaciren, Le, G. M., Li, A. F., Li, J. Y., Lou, Y.-Q., Lu, H., Lu, S. L., Meng, X. R., Mizutani, K., Mu, J., Munakata, K., Nagai, A., Nanjo, H., Nishizawa, M., Ohnishi, M., Ohta, I., Onuma, H., Ouchi, T., Ozawa, S., Ren, J. R., Saito, T., Saito, T. Y., Sakata, M., Sako, T. K., Shibata, M., Shiomi, A., Shirai, T., Sugimoto, 
H., Takita, M., Tan, Y. H., Tateyama, N., Torii, S., Tsuchiya, H., Udo, S., Wang, B., Wang, H., Wang, X., Wang, Y., Wang, Y. G., Wu, H. R., Xue, L., Yamamoto, Y., Yan, C. T., Yang, X. C., Yasue, S., Ye, Z. H., Yu, G. C., Yuan, A. F., Yuda, T., Zhang, H. M., Zhang, J. L., Zhang, N. J., Zhang, X. Y., Zhang, Y., Yizhang, Zhaxisangzhu, Zhou, X. X., \& Tibet AS $\gamma$ Collaboration. 2011, Advances in Space Research, 47, 629

Tinbergen, J. 1982, A\&A, 105, 53

Vallerga, J. 1998, ApJ, 497, 921

Vallerga, J. \& Slavin, J. 1998, in Berlin Springer Verlag Lecture Notes in Physics, The Local Bubble and Beyond, Vol. 506, 79-82

Vergely, J., Valette, B., Lallement, R., \& Raimond, S. 2010, A\&A, 518, A31+

Vernetto, S., Guglielmotto, Z., Zhang, J. L., \& for the ARGO-YBJ Collaboration. 2009, ArXiv e-prints

Wiktorowicz, S. J. \& Nofi, L. A. 2015, ApJ, 800, L1

Wiktorowicz, S. J., Nofi, L. A., Jontof-Hutter, D., Kopparla, P., Laughlin, G. P., Hermis, N., Yung, Y. L., \& Swain, M. R. 2015, ArXiv e-prints, 1507.03588

Wisniewski, J. P., Bjorkman, K. S., Magalhães, A. M., Bjorkman, J. E., Meade, M. R., \& Pereyra, A. 2007, ApJ, 671, 2040

Wolleben, M. 2007, ApJ, 664, 349

Wood, B., Redfield, S., Linsky, J., Müller, H.-R., \& Zank, G. 2005, ApJS, 159, 118

Wood, B. E., Redfield, S., Linsky, J. L., \& Sahu, M. S. 2002, ApJ, 581, 1168 
Table 1. Interstellar Magnetic Field Directions

\begin{tabular}{|c|c|c|}
\hline Magnetic Field & $\begin{array}{l}\text { Merit }^{(A)} \\
\text { function }\end{array}$ & $\begin{array}{l}\text { ISMF Direction }^{(B)} \\
\quad \ell, b \text { (deg.) }\end{array}$ \\
\hline Paper I & Unweighted fit & $38,23( \pm 35)$ \\
\hline Paper II & $F_{\mathrm{II}}\left(\mathrm{B}_{\mathrm{i}}\right)$ & $47 \pm 15,25 \pm 20$ \\
\hline All stars $\left(B_{\mathrm{ALL}}\right)$ & $F_{\text {II }}\left(\mathrm{B}_{\mathrm{i}}\right)$ & $16.3,27.0( \pm 15)$ \\
\hline Interstellar ( $\mathrm{B}_{\mathrm{POL}}$, no filament stars) & $F_{\mathrm{II}}\left(\mathrm{B}_{\mathrm{i}}\right)$ & $36.2,49.0( \pm 16)$ \\
\hline Filament $\left(B_{\mathrm{FIL}} \text {, only filament stars }\right)^{(C)}$ & $F_{\mathrm{II}}\left(\mathrm{B}_{\mathrm{i}}\right)$ & $359.3,19.0( \pm 10.2)$ \\
\hline $\operatorname{IBEX}\left(\mathrm{B}_{\operatorname{IBEX}}\right)^{(D)}$ & & $34.8 \pm 4.3,56.6 \pm 1.2$ \\
\hline Angle between $\mathrm{B}_{\mathrm{POL}}$ and $\mathrm{V}_{\mathrm{CLIC}, \mathrm{LSR}}{ }^{(E)}$ & & $76.8(+23.5,-27.6)$ \\
\hline Angle between $\mathrm{B}_{\mathrm{POL}}$ and $\mathrm{B}_{\mathrm{IBEX}}$ & & $7.6(+14.9,-7.6)$ \\
\hline Angle between $\mathrm{B}_{\mathrm{IBEX}}$ and $\mathrm{V}_{\mathrm{LIC}, \mathrm{LSR}}{ }^{\mathrm{F}}$ & & $96.9 \pm 8.5$ \\
\hline
\end{tabular}

${ }^{(A)}$ Eqn. [1]

${ }^{(B)}$ The quantities $\ell, b$ are the direction of the ISMF in galactic coordinates.

${ }^{(C)}$ Direction of the ISMF traced the polarization filament, from Frisch et al. (2015a)

${ }^{(D)}$ ISMF direction traced by the IBEX Ribbon, $\mathrm{B}_{\mathrm{IBEX}}$, corresponding to the weighted mean of the energy-dependent center of the IBEX Ribbon arc at $\lambda=$ $219.2 \pm 1.3, \beta=39.9 \pm 2.3$ (Funsten et al. 2013).

${ }^{(E)}$ Based on an upwind direction for the LSR CLIC velocity vector of $\ell=335.6 \pm$ 13.4, $b=-7.0 \pm 9.0, V=-17.3 \pm 4.9$ (Appendix D).

${ }^{(F)}$ See Appendix D for the LSR velocity of the LIC. 
Table 2. Polarization data

\begin{tabular}{|c|c|c|c|c|c|}
\hline Star & $\begin{array}{c}\ell, b \\
(\mathrm{deg})\end{array}$ & $\begin{array}{c}\text { Distance } \\
\text { (pc) }\end{array}$ & $\begin{array}{l}\theta_{\mathrm{RA}}^{\mathrm{A}} \\
(\mathrm{deg})\end{array}$ & $\begin{array}{l}\text { Polarization } \\
\quad\left(10^{-5}\right)\end{array}$ & Source \\
\hline HIP98130 & $9,-27$ & 19 & $131.0 \pm 22.5$ & 23. \pm 23 . & LNA \\
\hline HIP11276 & $354,-61$ & 28 & $104.0 \pm 8.7$ & $32 . \pm 10$. & LNA \\
\hline HIP2790 & $316,-76$ & 29 & $157.0 \pm 12.1$ & 31. \pm 14 & LNA \\
\hline HIP10301 & $279,-59$ & 29 & $31.0 \pm 2.1$ & 124. \pm 9 & LNA \\
\hline HIP11197 & $14,-61$ & 26 & $128.0 \pm 29.5$ & $27 . \pm 45$ & LNA \\
\hline HIP95467 & $329,-28$ & 26 & $118.0 \pm 17.7$ & 24. \pm 17 . & LNA \\
\hline HIP90355 & 37,10 & 27 & $111.0 \pm 9.2$ & 120. \pm 40 . & LNA \\
\hline HD105330 & 292,31 & 33 & $95.0 \pm 25.9$ & 45. \pm 57. & LNA \\
\hline HD78351 & 258,9 & 39 & $30.0 \pm 10.4$ & 42. \pm 16 . & LNA \\
\hline HD111232 & $303,-5$ & 30 & $92.0 \pm 32.4$ & $30 . \pm 64$ & LNA \\
\hline HD128674 & 317,3 & 27 & $63.0 \pm 16.7$ & 73. \pm 48 & LNA \\
\hline HD177409 & $327,-27$ & 35 & $133.0 \pm 15.4$ & $37 . \pm 22$. & LNA \\
\hline HD117939 & 312,23 & 30 & $40.0 \pm 10.2$ & 78. \pm 29 & LNA \\
\hline HD125162 & 87,64 & 30 & $25.0 \pm 15.0$ & $2.72 \pm 1.45$ & KVA \\
\hline HD130109 & 355,52 & 39 & $45.2 \pm 8.3$ & $5.46 \pm 1.60$ & KVA \\
\hline HD132052 & 351,46 & 28 & $45.7 \pm 31.6$ & $1.88 \pm 2.32$ & KVA \\
\hline HD137391 & 60,56 & 37 & $24.5 \pm 22.3$ & $1.42 \pm 1.17$ & KVA \\
\hline HD175638 & 37,0 & 40 & $85.0 \pm 27.3$ & $2.10 \pm 2.16$ & KVA \\
\hline HD185395 & 82,13 & 19 & $67.1 \pm 8.7$ & $3.27 \pm 1.00$ & KVA \\
\hline HD19373 & $144,-7$ & 11 & $25.2 \pm 8.7$ & $4.04 \pm 1.24$ & KVA \\
\hline HD91889 & 258,38 & 25 & $64.6 \pm 19.1$ & $5.09 \pm 3.52$ & KVA \\
\hline HD124850 & 337,51 & 21 & $148.5 \pm 23.0$ & $1.38 \pm 1.17$ & KVA \\
\hline HD14055 & $142,-25$ & 36 & $7.9 \pm 4.4$ & $4.54 \pm 0.69$ & KVA \\
\hline HD15335 & $146,-28$ & 31 & $21.4 \pm 14.9$ & $4.28 \pm 2.28$ & KVA \\
\hline HD18256 & $160,-35$ & 35 & $28.2 \pm 12.4$ & $1.71 \pm 0.75$ & KVA \\
\hline HD18404 & $158,-33$ & 32 & $27.0 \pm 14.1$ & $1.03 \pm 0.52$ & KVA \\
\hline HD206901 & $78,-20$ & 35 & $6.9 \pm 36.9$ & $0.82 \pm 1.23$ & KVA \\
\hline HD222603 & $90,-56$ & 31 & $21.7 \pm 37.3$ & $1.17 \pm 1.78$ & KVA \\
\hline HD25490 & $184,-33$ & 40 & $26.9 \pm 19.2$ & $2.24 \pm 1.56$ & KVA \\
\hline HD25570 & $182,-31$ & 36 & $24.5 \pm 10.9$ & $2.59 \pm 1.00$ & KVA \\
\hline HD8829 & $155,-73$ & 36 & $17.8 \pm 29.7$ & $2.20 \pm 2.51$ & KVA \\
\hline HD13555 & $147,-37$ & 30 & $11.3 \pm 13.8$ & $2.45 \pm 1.20$ & KVA \\
\hline
\end{tabular}

${ }^{A}$ Polarization position angles are presented in equatorial coordinates.

${ }^{\mathrm{B}}$ The polarizations represent the fractional linear polarizations of the 
E-component of the starlight

${ }^{C}$ The data source refers to the telescope where the data were acquired. The KVA data were acquired by A. Berdyugin and V. Piirola using BVR filters. The LNA data were acquired by A. M. Magalhaes and D. B. Seriacopi using the $\mathrm{V}$ filter. 


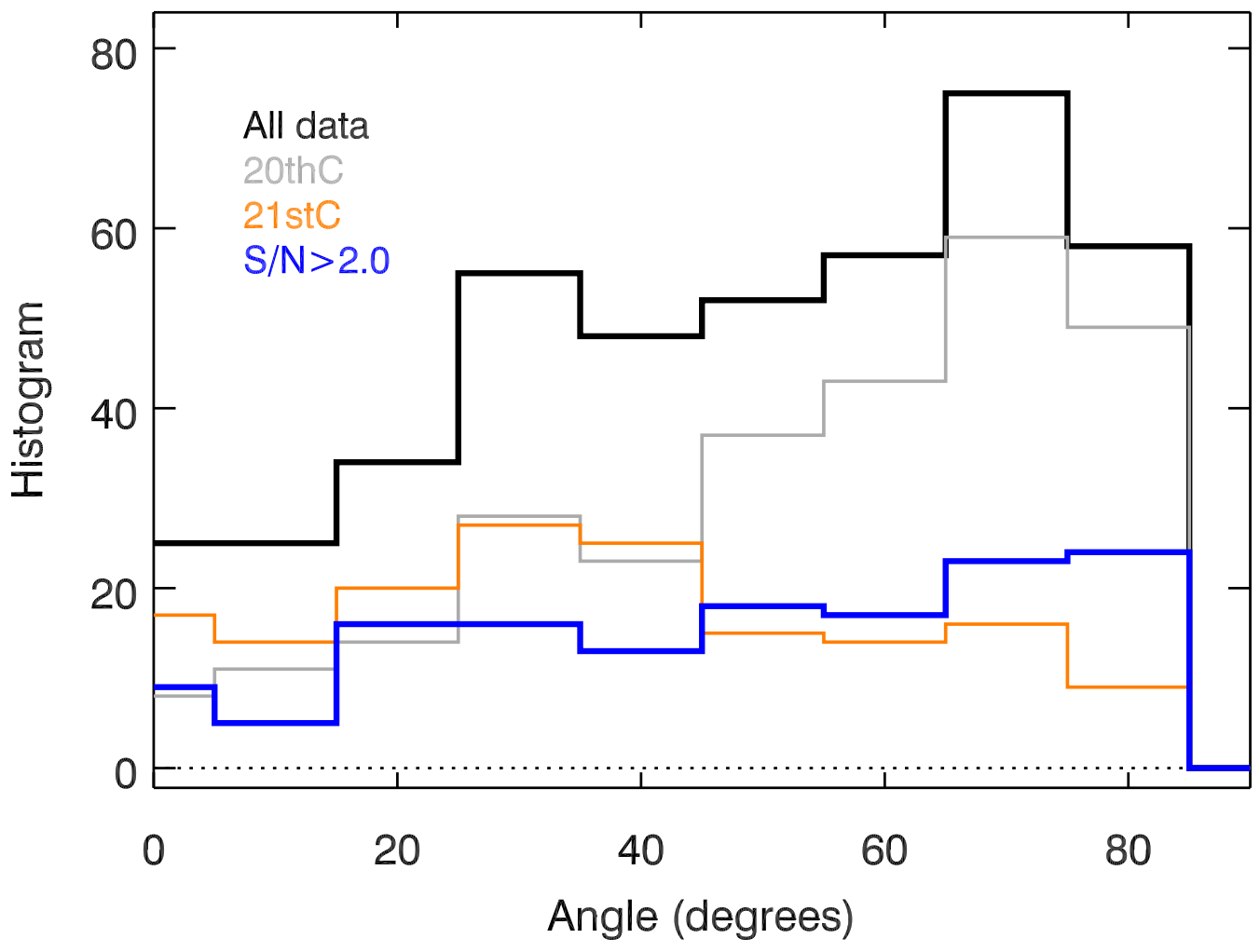

Fig. 1.- Angular distribution of stars with respect to heliosphere nose direction. The number of stars in the designated category is plotted against the angle between the star and the heliosphere nose. The thin gray and orange lines represent data collected in the 20th and 21st centuries, respectively. The smaller number of 21th century targets at large angles from the heliosphere nose, compared to the same numbers for 20st century stars, is partly due to choices made in the selection of the target stars (Table 21). The black line shows the total data set. The blue line shows the subsample with $P / \Delta P \geq 2.0$. 


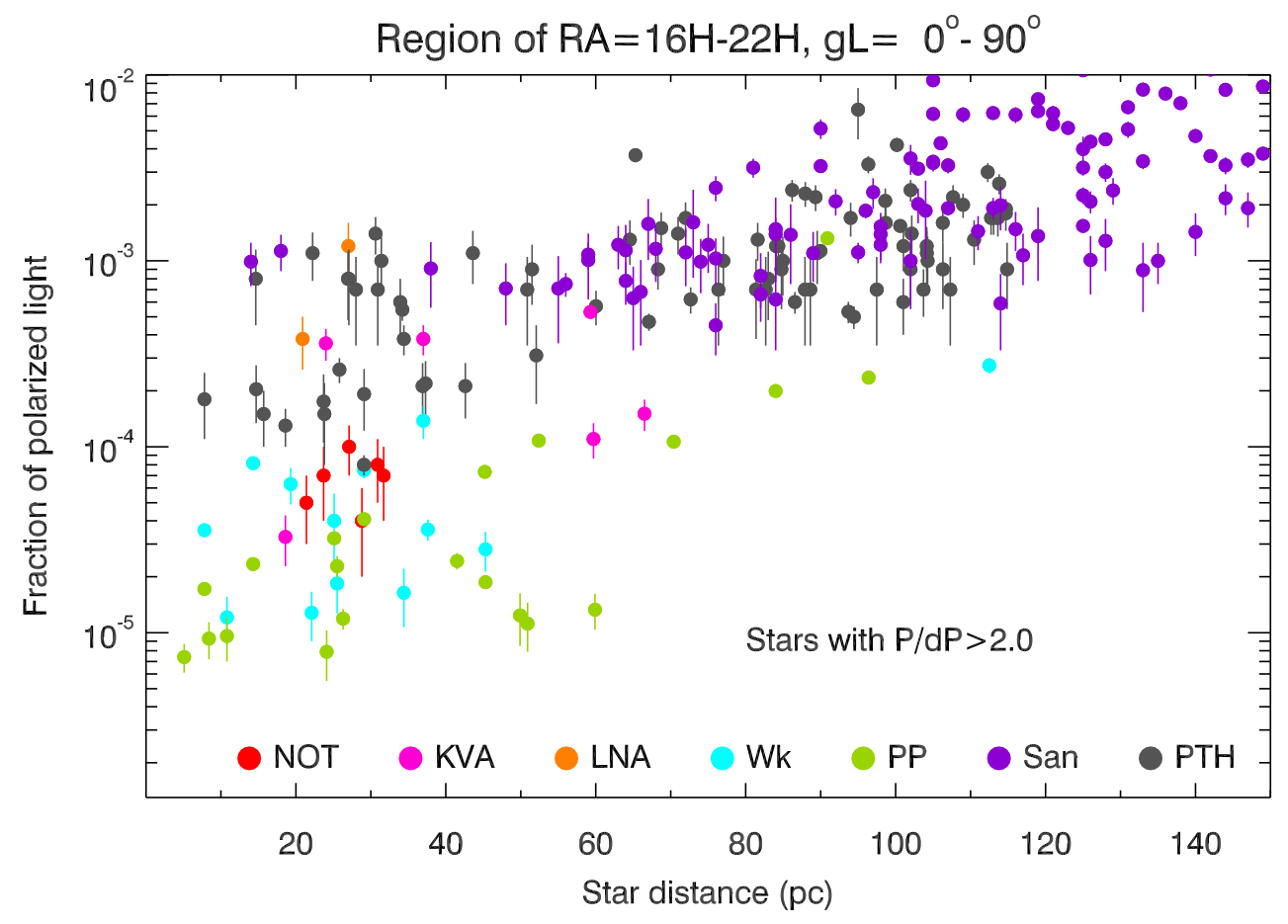

Fig. 2.- Polarization strengths versus distance. The fractional linear polarizations of the Ecomponent of starlight is plotted for stars in the first galactic quadrant, $\ell=0^{\circ}-90^{\circ}$, between right ascensions $17 \mathrm{HR}$ and $22 \mathrm{HR}$, and where $P / \Delta P>2.0$. The general increase of polarization strengths with distance becomes less obvious near the Sun where clumping of gas and dust becomes evident, and systematic differences due to instrumental sensitivities are relatively more important. These data create a heterogeneous set collected using polarimeters with different bandpasses and sensitivity levels. Color coding denotes the data source: KVA (red), NOT (pink, Paper II), 20th century data (PT, gray, Piirola 1977; Tinbergen 1982; Heiles 2000), LNA (orange), Lick (turquoise, Wiktorowicz et al. 2015), PlanetPol (green, Bailey et al. 2010), and (Santos et al. 2011, purple). The distance uncertainties (not shown) are typically less than $4 \%$ of the star distances. 
Table 3. Summary of LSR vectors and directions

\begin{tabular}{lc}
\hline \hline Quantity & $\begin{array}{c}\text { LSR Vector Velocity } \\
\ell(\mathrm{deg}), b(\mathrm{deg}), \mathrm{V}\left(\mathrm{km} \mathrm{s}^{-1}\right)\end{array}$ \\
\hline Vector velocity of solar apex motion $^{\mathrm{A}}$ & $47.8 \pm 2.9,23.8 \pm 2.0,18.0 \pm 0.9$ \\
Vector velocity of bulk CLIC LSR motion $^{\mathrm{B}}$ & $155.6 \pm 13.4,7.0 \pm 9.0,17.3 \pm 4.9$ \\
Vector velocity of bulk LIC LSR motion $^{\mathrm{C}}$ & $141.1 \pm 5.9,2.4 \pm 4.2,17.2 \pm 1.9$ \\
\hline
\end{tabular}

${ }^{A}$ Based on the U,V,W components of the solar apex motion in Schönrich et al. $(2010)$.

${ }^{\mathrm{B}}$ Based on the heliocentric velocity vector of the CLIC derived in Frisch et al. (2002), with the additional assumption that uncertainties on the longitude and latitude of the vector are $2^{\circ}$ each.

${ }^{\mathrm{C}}$ This LSR velocity for the LIC is based on the heliocentric velocity derived for the flow of interstellar $\mathrm{He}^{\circ}$ through the heliosphere that compared in situ IBEX-LO $\mathrm{He}^{\circ}$ data with multivariate simulations of the particle trjacetories (Schwadron et al. 2015). 


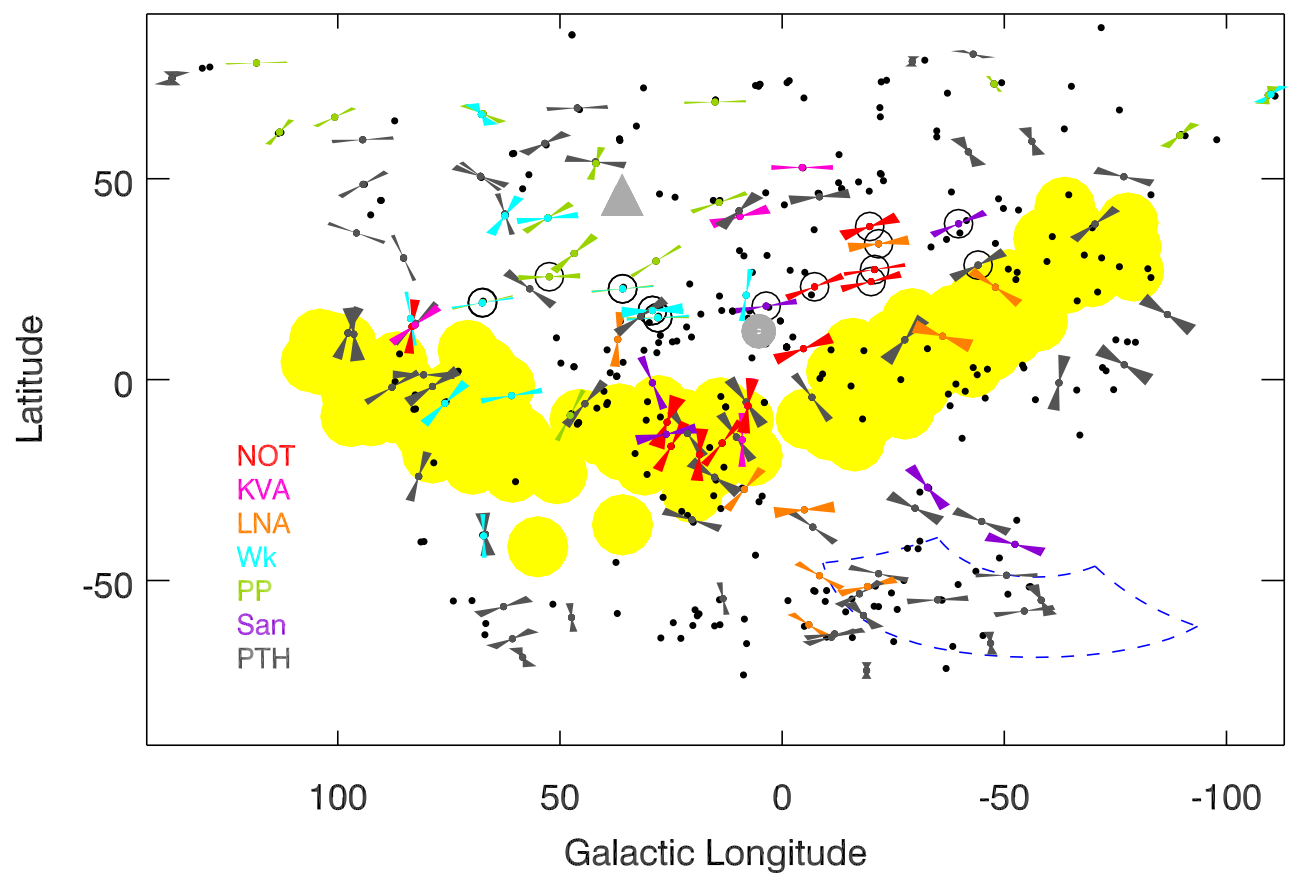

Fig. 3.- Polarization position angles for stars with $P / \Delta P>2.0$ and within $40 \mathrm{pc}$ and $90^{\circ}$ of the heliosphere nose are plotted. The fan-shaped polygons indicate the angular uncertainty of the polarization position angles $\Delta \theta$. Stars with $P / \Delta P<2 \sigma$ are plotted with dots. The regions of the highest fluxes of $1 \mathrm{keV}$ ENAs, corresponding to the IBEX Ribbon, are denoted in yellow. Circled stars indicate stars that trace the polarization filament $(\$ 4.2, \$ 5.3$ Frisch et al. 2015a). The directions of the best-fitting ISMF $\mathrm{B}_{\mathrm{POL}}$ and heliosphere nose are shown as gray triangles and dots, respectively. The dashed lines enclose the region observed by BICEP for CMB B-mode polarization (\$5.6). Color coding of the polarizations indicate the data source (see Fig. 2). 

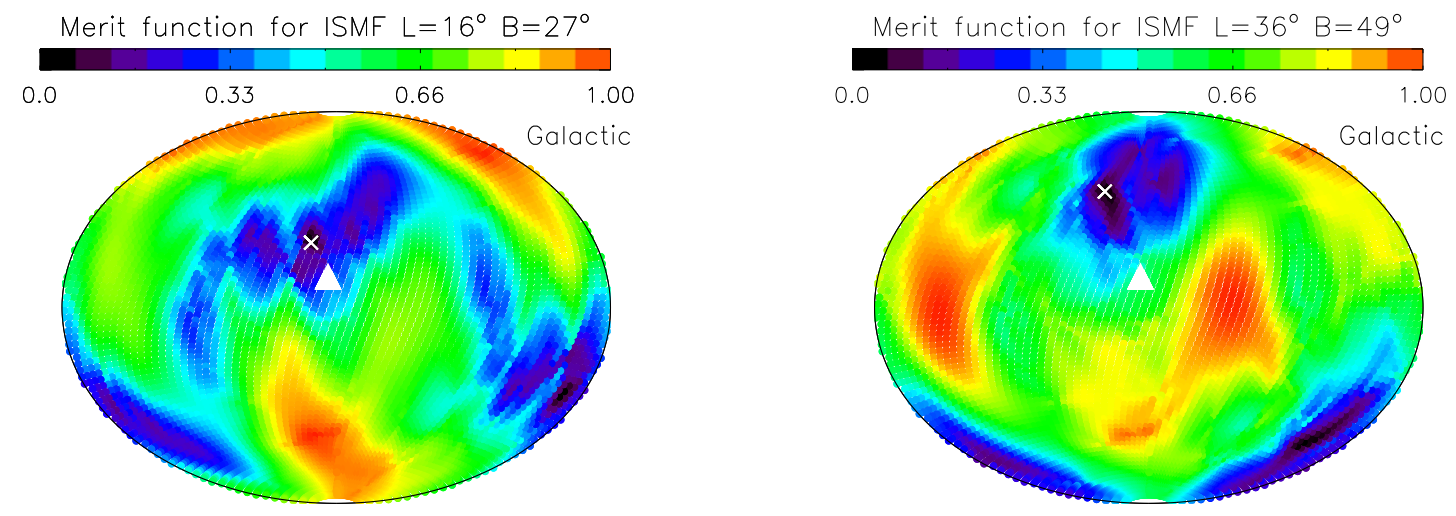

Fig. 4.- Values of the merit function, $F_{\mathrm{II}}\left(\mathrm{B}_{\mathrm{i}}\right)$ for each location on the sky. $F_{\mathrm{II}}\left(\mathrm{B}_{\mathrm{i}}\right)$ is normalized to the minimum value that gives the best-fitting ISMF direction. The color scale is based on a log scale. Left: The merit function for the fit to eqn. 1 that utilizes all qualifying polarization measurements. The best-fitting ISMF direction for this star sample, $B_{\mathrm{ALL}}$, is toward $\ell, b=16.3^{\circ}, 27.0^{\circ}$. Right: The merit function calculated by omitting the filament stars from the fitted sample. The best-fitting ISMF without the filament stars, $\mathrm{B}_{\mathrm{POL}}$, is toward $\ell=36.2^{\circ}, b=49.0^{\circ}$. The location of the minimum of $F_{\mathrm{II}}\left(\mathrm{B}_{\mathrm{i}}\right)$, is plotted with an "X", and the heliosphere nose is located at the triangle. Uncertainties on these directions are shown in Fig. 5 and listed in Table 1. The figures are centered on the galactic center, with galactic longitude increasing toward the left and latitude increasing toward the top. 

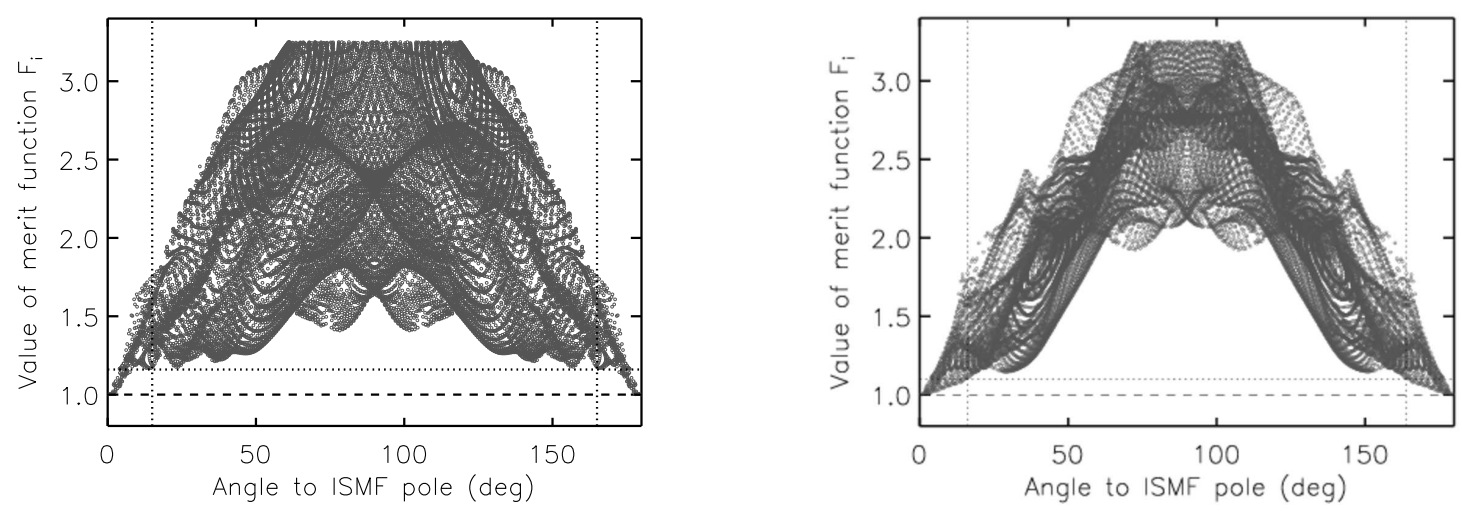

Fig. 5.- Uncertainties on the best-fitting ISMF direction for $B_{\mathrm{ALL}}$ (left) and $\mathrm{B}_{\mathrm{POL}}$ (right). The uncertainties are given by the angular distribution normalized merit function $F_{\mathrm{II}}\left(\mathrm{B}_{\mathrm{i}}\right)$ values (vertical axis), which are plotted against the angle between the best-fitting ISMF direction and the merit function at each point on the sky (horizontal axis). By definition, the merit function minimum is located at the position of the best-fitting ISMF. For $B_{\text {ALL }}$ the uncertainties on the best-fitting ISMF are defined as the first minimum in the merit function array at $\pm 15^{\circ}$ (vertical dotted line, left figure). For $B_{\mathrm{POL}}$, the uncertainty is arbitrarily assigned to the angle where the merit function is $10 \%$ above the best-fitting value, or $\pm 16^{\circ}$. The values of $f_{\mathrm{n}}\left(B_{\mathrm{i}}\right)$ for the star sample with the filament stars omitted (right) has a more compact angular distribution and is better defined than the function that includes the entire polarization data set (left, see Fig. 41). 

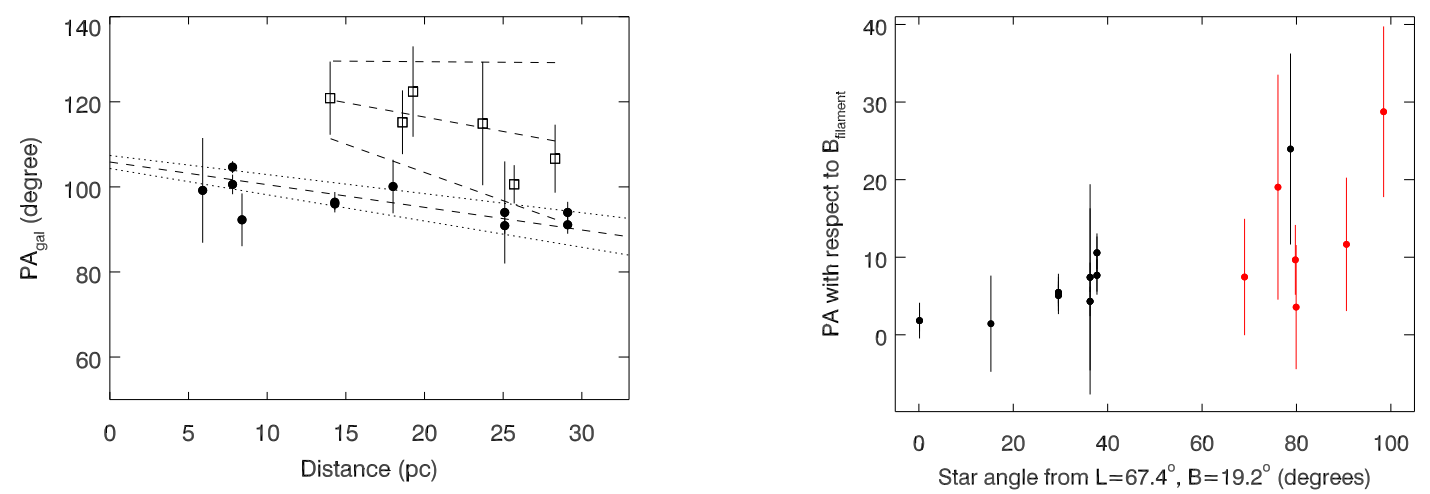

Fig. 6.- Left: Galactic polarization position angles are plotted against the star distance for the two sets of stars that make up the magnetic 'filament' feature (\$4.2). Both sets of stars show polarization position angles expressed in galactic coodinates, $\theta_{\text {gal }}$, that rotate with the distance of the star. The separate linear fits performed to the two subsets of stars in this filament are shown, together with the $1 \sigma$ uncertainty of the fits. Right: The polarization position angles (vertical axis) are plotted against the angular distance from the star HD 172167 that is located at the end of the filament (horizontal axis). The position angles are expressed relative to the direction of the ISMF that provides the best fit to the filament polarizations, $B_{\mathrm{FIL}}$. The ISMF derived from filament polarizations provides a more uniform description of polarization position angles than does the north galactic pole. 


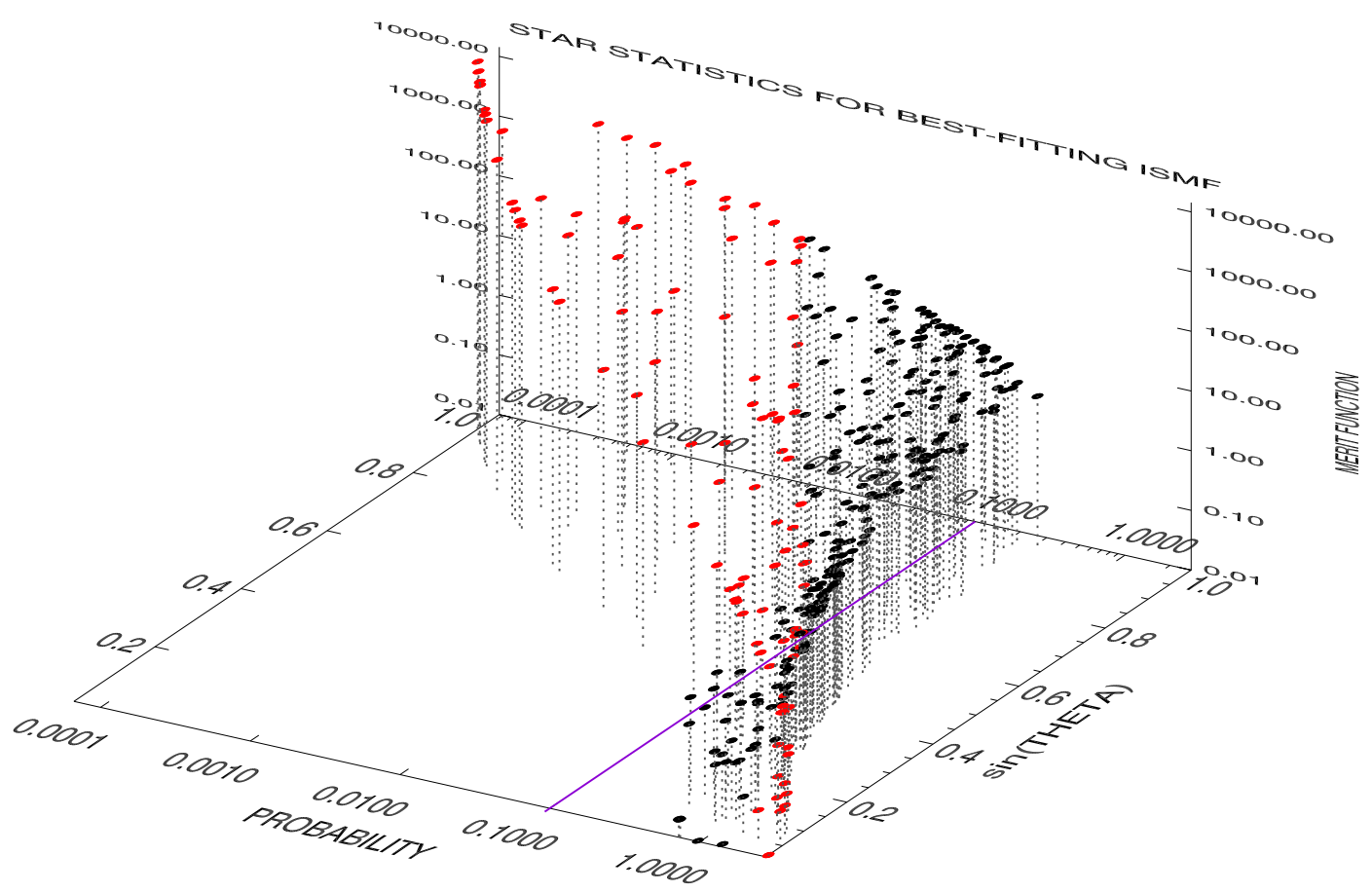

Fig. 7.- Distribution of merit function components in 3D. The value of the merit function $F_{\mathrm{II}}\left(\mathrm{B}_{\mathrm{i}}\right)$ for each star determined with respect to $\mathrm{B}_{\mathrm{POL}}$, the best-fitting ISMF direction, is shown. The front horizontal axis shows the normalized probability, $G_{\mathrm{n}}$, that the observed polarization position angle is equal to the expected angle for $\mathrm{B}_{\mathrm{POL}}$ (eqn. 2). The right horizontal axis "sin(theta)" is the sine of $\theta_{\mathrm{PA}}$ for each star in the coordinate system defined for $\mathrm{B}_{\mathrm{POL}}$ located at the pole of the system. The vertical axis, "merit function" $\left(f_{\mathrm{n}}\left(B_{\mathrm{i}}\right)\right)$ gives the merit function for each star (eqn. 1). Red points indicate stars with $P / \Delta P>2.0$. This figure shows that the minimization method used to select out the best-fitting ISMF direction (§3) is sensitive to stars in the front-right hand corner of the figure, where the values of the merit function being minimized are small, and those in the rear left corner, where the measurement errors are small but the statistical probability that the value corresponds to the true angle represented by the best-fit is small. The stars in the rear left corner are candidates for tracing a new component of the local ISMF structure 


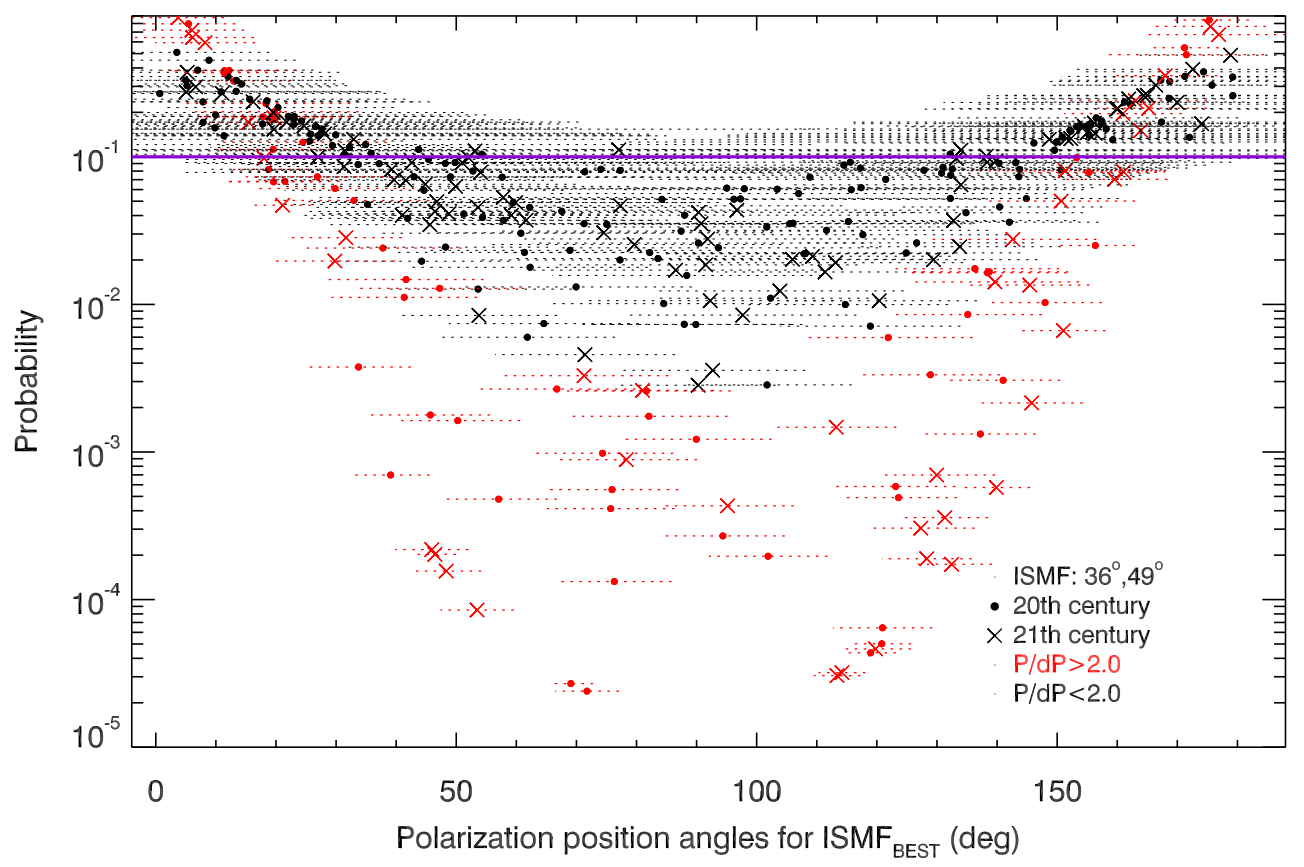

Fig. 8. - Statistical probabilities for individual stars. Statistical properties of the stars with respect to the best-fitting ISMF direction $\mathrm{B}_{\mathrm{POL}}$ are plotted. The horizontal axis shows the polarization position angle in the rotated coordinate frame corresponding to the best-fitting ISMF direction $B_{\mathrm{POL}}$ (Table 1). The vertical axis gives the statistical probability (eqn. 2 of the data point. The red (black) points represent stars with polarization strengths of $P / \Delta P$ larger (smaller) than 2.0. Data collected during the 20th and 21th centuries are coded as "dots" and "crosses", respectively. Polarization position angles that are perfectly aligned with $\mathrm{B}_{\mathrm{POL}}$ have position angles of $0^{\circ}$ or $180^{\circ}$. For reference, the purple line shows the same probability level as the purple line in Fig. 7. 


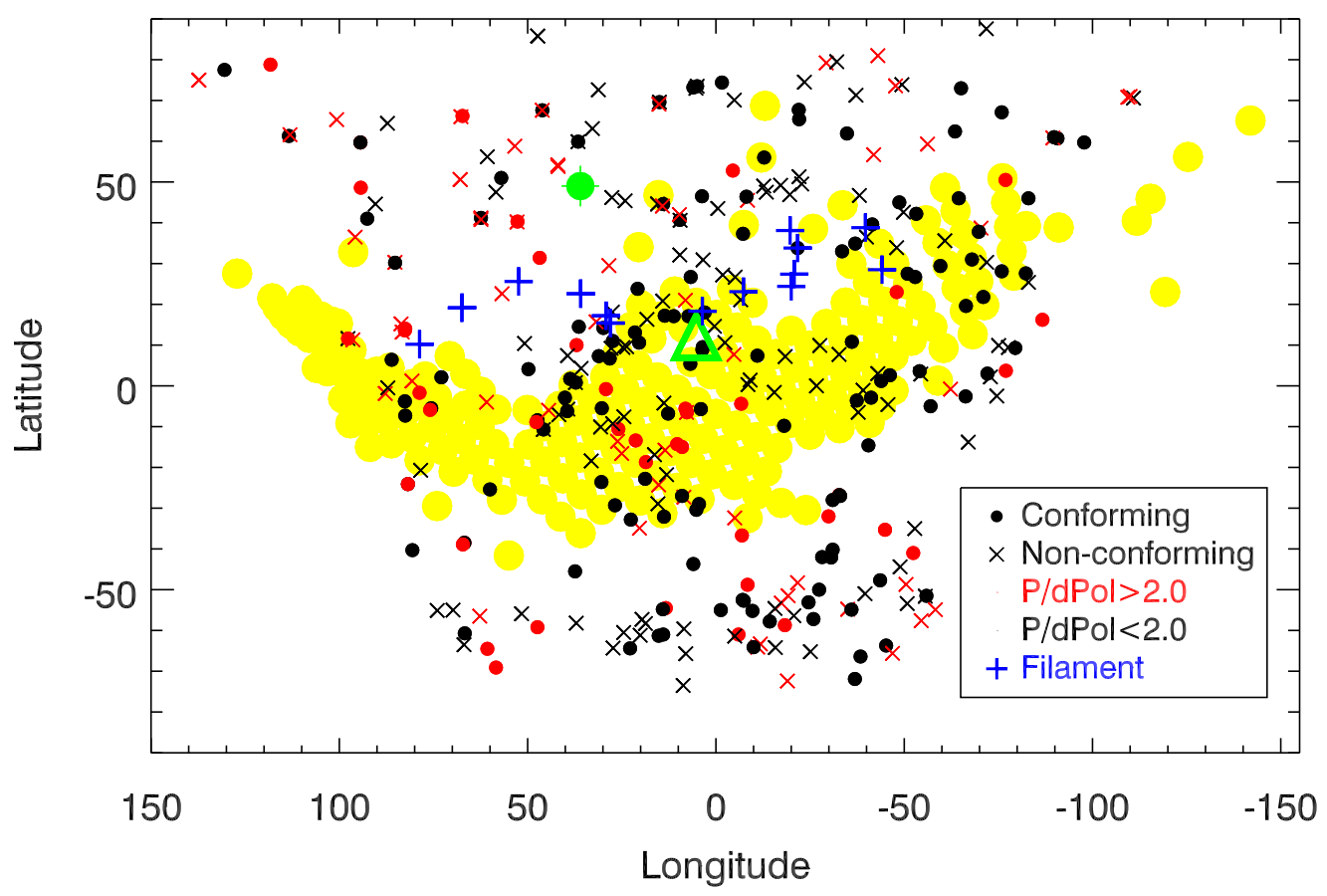

Fig. 9.- Stars used in the study are plotted with coding that indicates whether or not the polarization position angles agree with the ISMF field direction $\mathrm{B}_{\mathrm{POL}}$. The galactic locations of the stars in Fig. 7 are plotted and coded by the value of $F_{\mathrm{II}}\left(\mathrm{B}_{\mathrm{i}}\right)$ and $P / \Delta P$. The star set is divided into two equally sized groups, defined by stars that are in the top or bottom half of stars with polarization position angles that have polarization position angles that agree with the direction $\mathrm{B}_{\mathrm{POL}}$. Filled circles show the half of the data that best comply with $\mathrm{B}_{\mathrm{POL}}$, with stars having polarization mean errors of $>2.0$ plotted in red, and the less significant data points plotted in black. The stars with position angles that do not match $\theta_{\mathrm{POL}}$ are plotted with with "X's". The distribution of position angles that do or do not agree with $\mathrm{B}_{\mathrm{POL}}$ are determined using the median value 3.54 of $F_{\mathrm{II}}\left(\mathrm{B}_{\mathrm{i}}\right)$. The green triangle and green dot show the locations of the heliosphere nose, and the best-fitting ISMF $\mathrm{B}_{\mathrm{POL}}$, respectively. The blue crosses show the locations of the filament stars. Note that the stars which best trace the same ISMF as the IBEX ribbon tend to be more concentrated near the heliosphere nose and at lower galactic latitudes. 


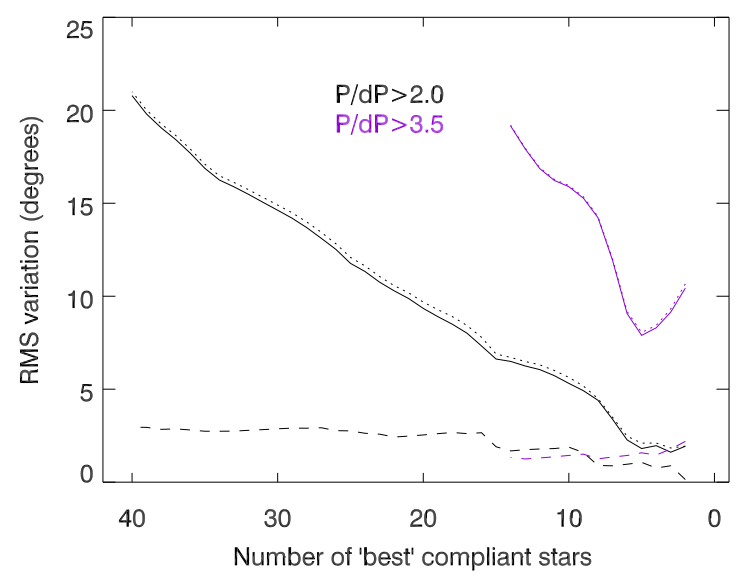

Fig. 10. - Component of the position angle dispersion that can be attributed to interstellar turbulence. The three quantities, interstellar turbulence, $\Phi_{\text {IS }}$ (solid lines), position angles $\theta_{\mathrm{POL}}$ relative to the best-fitting ISMF direction $\mathrm{B}_{\mathrm{POL}}$ (dotted lines), and mean measurement errors $\delta \theta_{\text {me }}$ (dashed lines) are displayed (see eqn. 44). Interstellar turbulence is evaluated both for the data subset consisting of 21 st century measurements and $P / \mathrm{dPol}>2.0$ (black), and $P / \mathrm{dPol}>3.5$ (purple). The ordering of stars along the horizontal axis is according to the $\theta_{\mathrm{POL}}$, with the stars that best-agree with $\mathrm{B}_{\mathrm{POL}}$ on the right of the horizontal axis, for the $P / \Delta P$ limits above. All positions on the lines represent results obtained by successively omitting the left-most (i.e. less compliant with $B_{\mathrm{POL}}$ ) stars from the calculation of $\Phi_{\mathrm{IS}}$. The minimum for the $P / \mathrm{dPol}>3.5$ data brackets the best approximation for the interstellar magnetic turbulence indicated by these data, $\Phi_{\mathrm{IS}} \sim 9^{\circ} \pm 1^{\circ}$. 


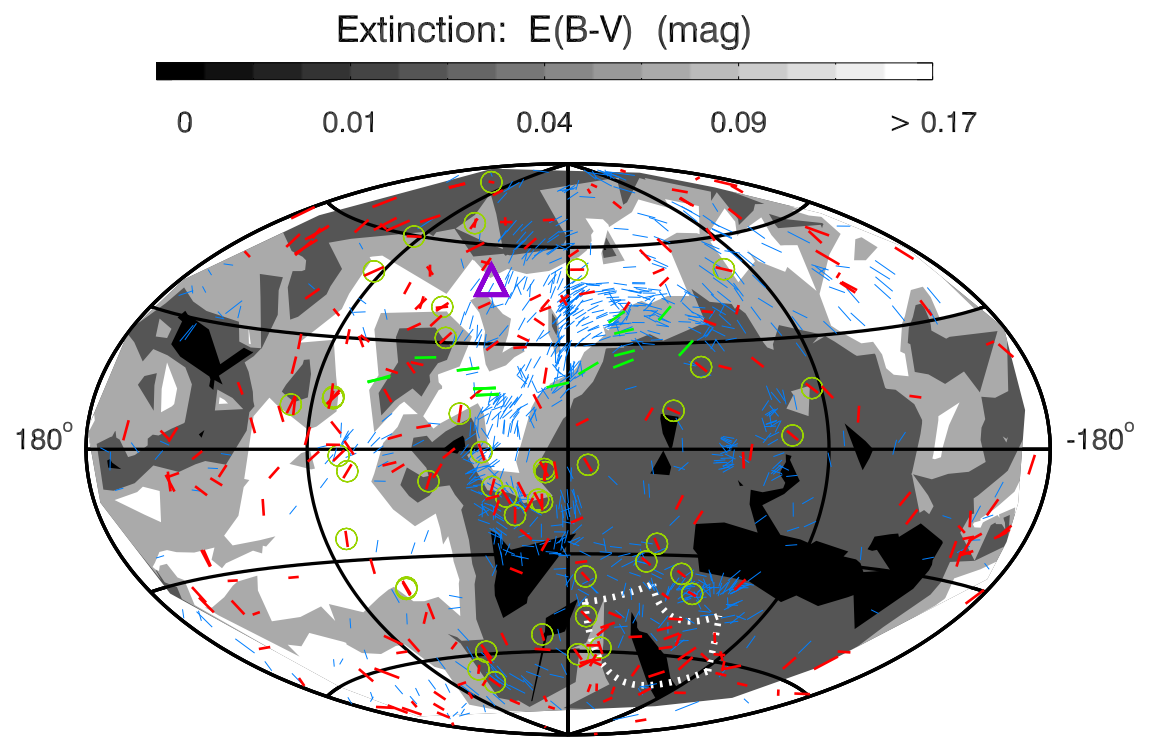

Fig. 11. - The amount of reddening of nearby stars is compared to polarized starlight. Reddening is represented by the cumulative smoothed color excess $\mathrm{E}(\mathrm{B}-\mathrm{V})$ of stars within 100 pc (Appendix B). The polarizations of stars within 40 pc are plotted with red bars, except that the filament polarizations are plotted with green bars. Nearby stars with polarization position angles that are in the best agreement with $\mathrm{B}_{\mathrm{POL}}$ have green circles around them (see text). The polarizations of stars within $300 \mathrm{pc}$ and that define Loop I are plotted with blue bars. The polarization patterns for the distant and nearby stars are similar in many regions suggesting that they are sampling a common magnetic field direction that is ordered by Loop I. The dominant nearby dust structure (lightest coloring) surrounds a cavity of low extinction centered below the galactic plane in the fourth galactic quadrant (central dark region). Loop I models suggest the dust structure is from the expansion of Loop I into the Local Bubble. The polarizations of southern hemisphere stars are more likely to trace $\mathrm{B}_{\mathrm{POL}}$ than northern hemisphere stars. The ISMF in the region tested by BICEP2 for the B-mode polarizations of the $\mathrm{CMB}$ (dotted lines) is different from $\mathrm{B}_{\mathrm{POL}}$. The direction of $\mathrm{B}_{\mathrm{POL}}$ is shown by the purple triangle. 


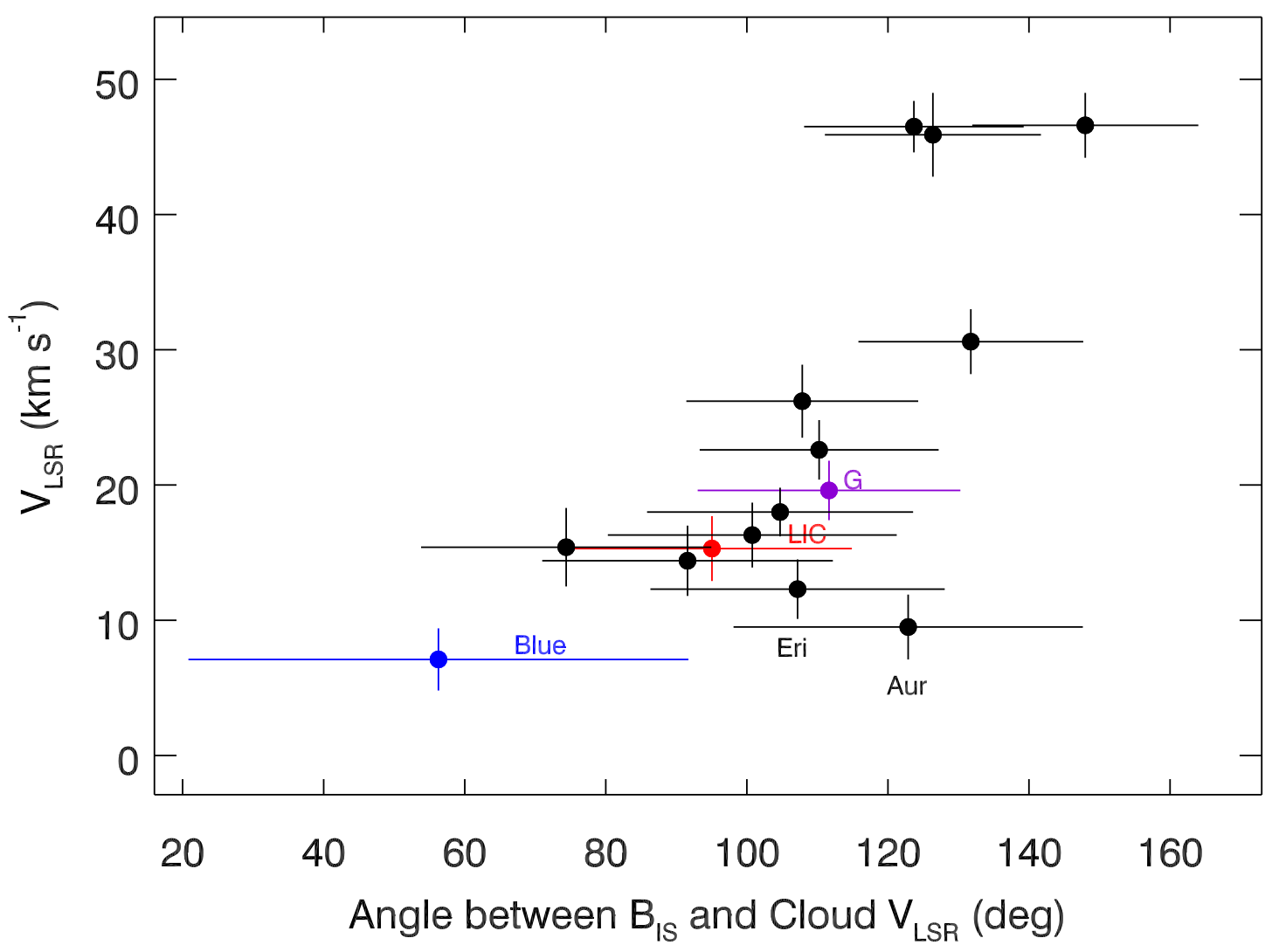

Fig. 12.- The LSR velocities of the fifteen cloud model for the CLIC (see text) are plotted against the angle between the LSR velocity and the magnetic field direction $\mathrm{B}_{\mathrm{POL}}$. The LSR velocity increases with the angle between $V_{\mathrm{LSR}}$ and $\mathrm{B}_{\mathrm{POL}}$, except for the labeled Eri and Aur clouds. Over half of the clouds travel at an angle that is quasi-perpendicular to $\mathrm{B}_{\mathrm{POL}}$ at angles $90^{\circ}-120^{\circ}$. Filled circles show clouds centered within $90^{\circ}$ of the heliosphere nose and the LIC is plotted in red. 


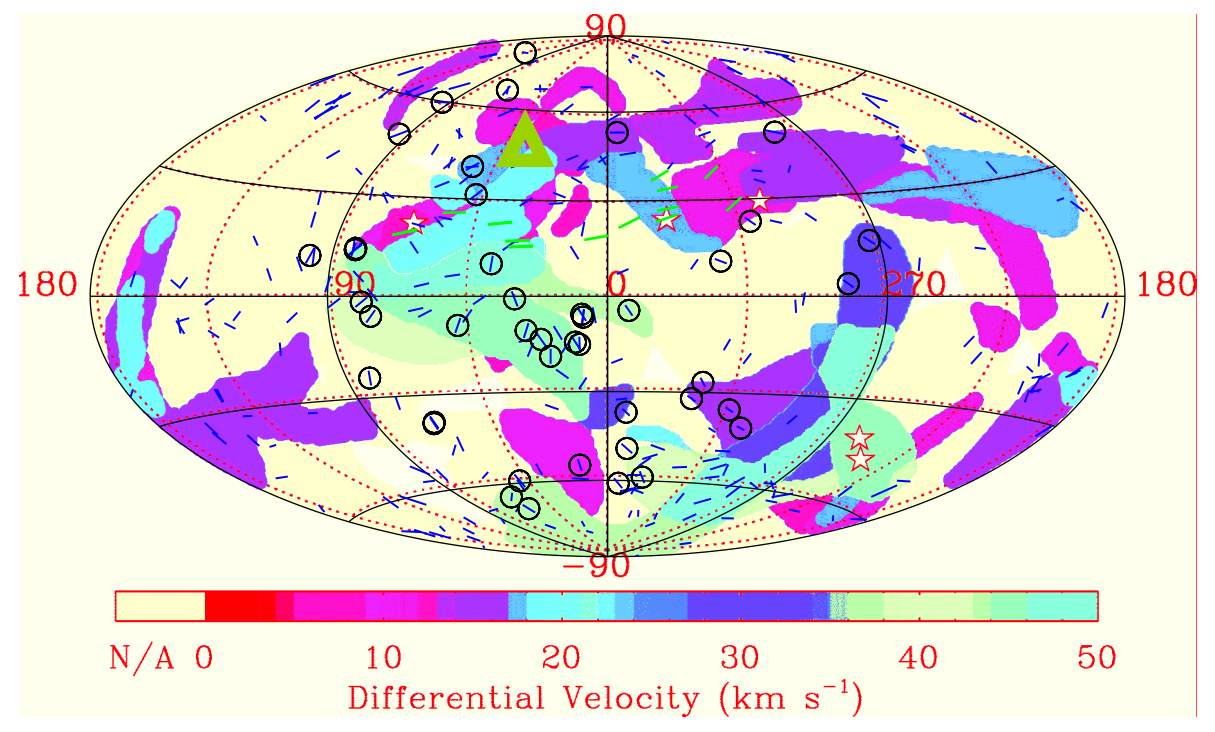

Fig. 13. - The polarizations of stars with mean errors $>2 \sigma$ are plotted on a representation of the differential velocities between clouds in the same sightline. The underlying velocity differential figure is a recolored version of Fig. 2 in Linsky et al. (2008). High differential velocities suggest colliding clouds. The polarizations of stars that conform to $\mathrm{B}_{\mathrm{POL}}$ are circled. The polarization vectors are shown for stars with $P / \Delta P>2.0$, and color coded with non-filament/filament polarizations in blue/green. Circled stars correspond to compliant stars in Fig. 11, $\mathrm{B}_{\mathrm{POL}}$ is found in the directions of clouds with velocities that span the entire range of differential velocity space from $0-50 \mathrm{~km} \mathrm{~s}^{-1}$, but favors locations in the first galactic quadrant. 


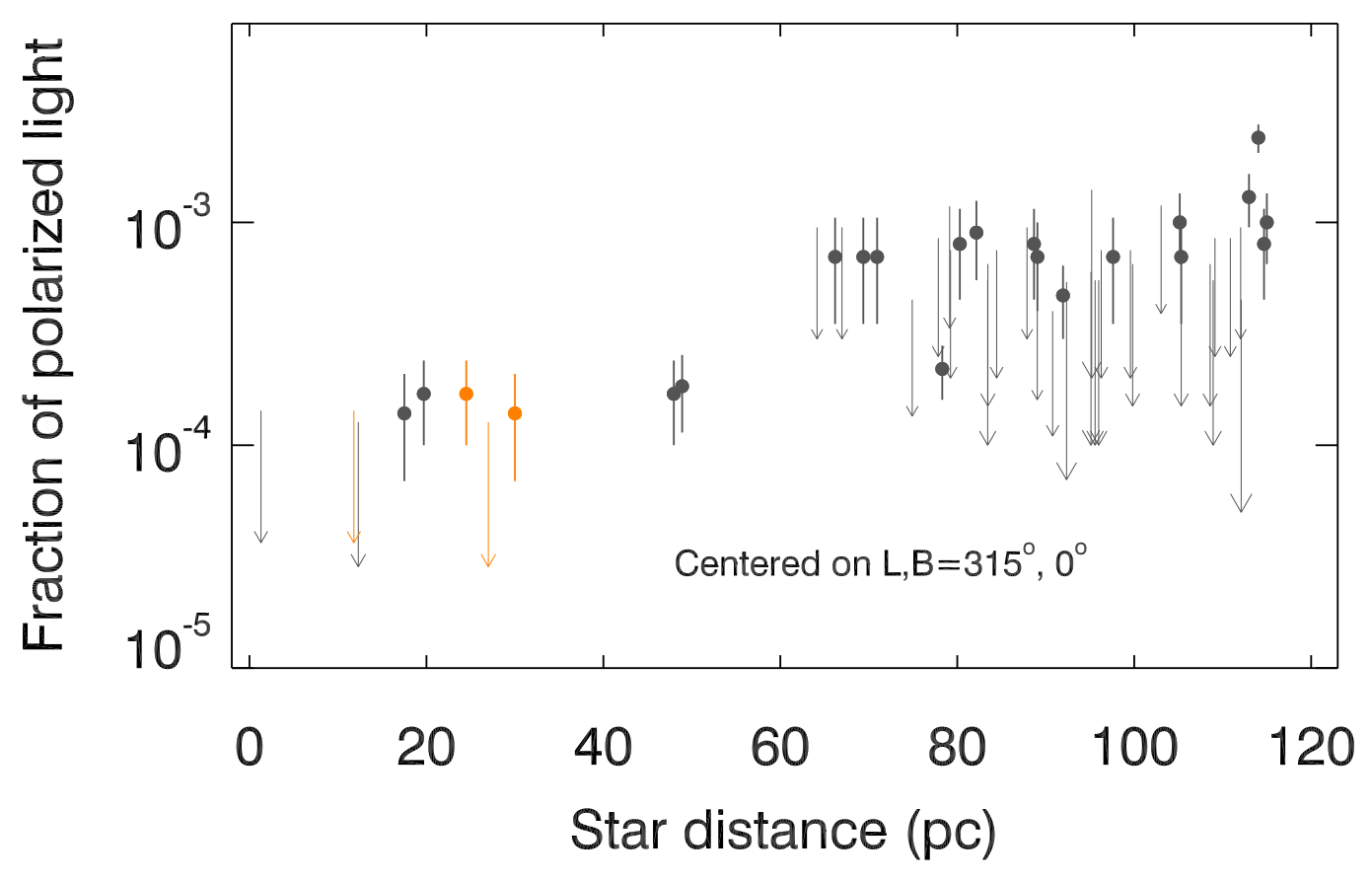

Fig. 14. - Polarizations are plotted for stars within $20^{\circ}$ of the nominal center of the G-cloud at $\ell, b=315^{\circ}, 0^{\circ}$ (according to the model of Redfield \& Linsky 2008). Two layers of polarizing grains are seen, at $\sim 19 \mathrm{pc}$ and $\sim 60 \mathrm{pc}$. The arrows show upper limits on polarizations $P / \Delta P<2.0$. 


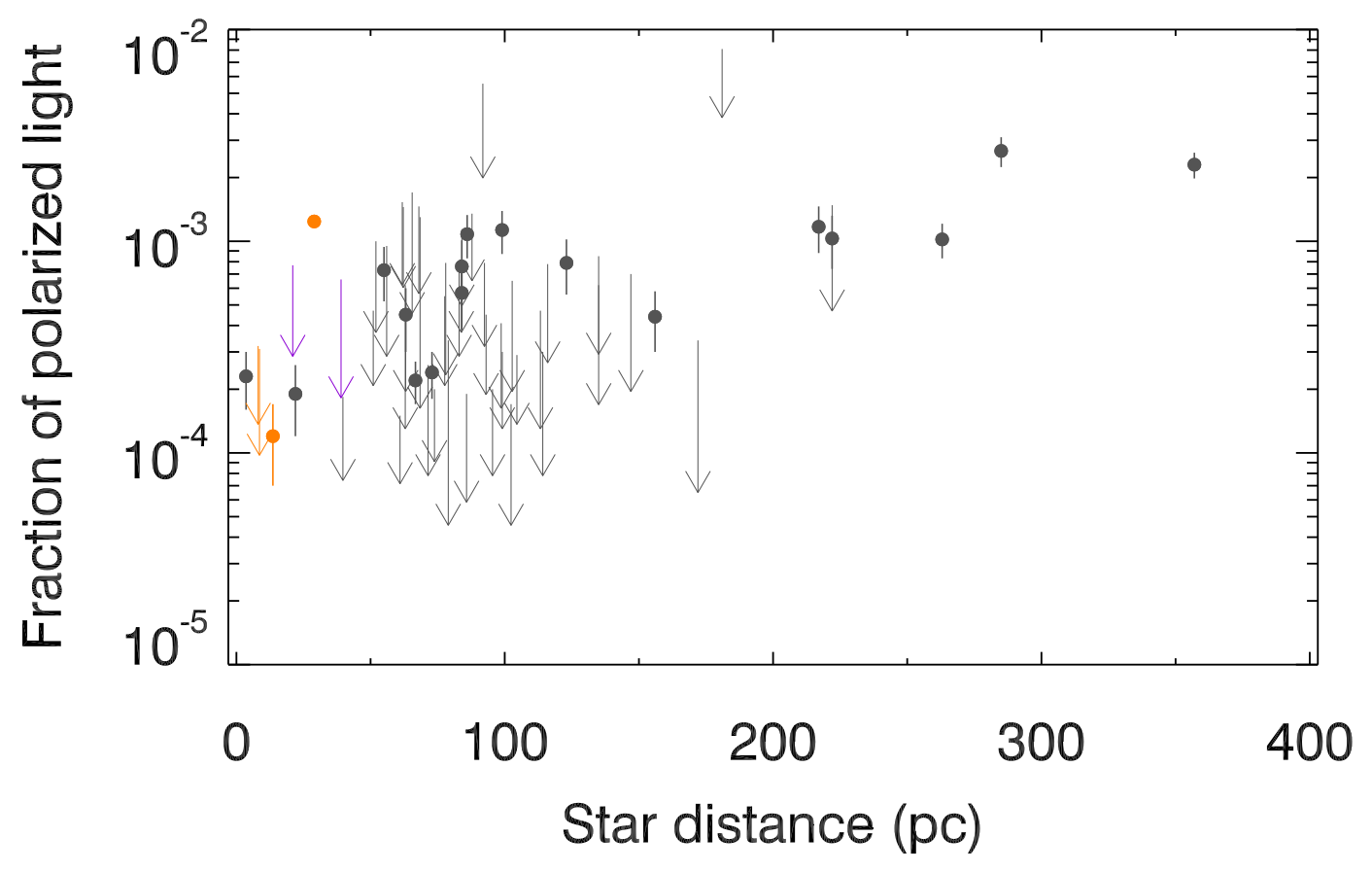

Fig. 15. - Polarizations of stars located in the BICEP2 field are plotted against distance. The observed polarizations suggest that the opacity in the $\mathrm{B}-\mathrm{V}$ band in this field is $A_{\mathrm{V}} \sim 0.069$ mag or more, depending on foreground depolarization. The two nearby stars with detected polarizations in the BICEP2 field and observed by LNA are HD 211415 and HIP 10301 (see symbol color-coding of Fig. [3). 